\title{
A Dynamic Model of Player Level-Progression Decisions in Online Gaming
}

\author{
Forthcoming Management Science \\ September 2021
}

\author{
Yi Zhao - Georgia State University \\ Sha Yang - University of Southern California \\ Matt Shum - California Institute of Technology \\ Shantanu Dutta - University of Southern California
}

\begin{abstract}
A key feature of online gaming, which serves as an important measure of consumer engagement with a game, is level progression, wherein players make play-or-quit decisions at each level of the game. Understanding users' level progression behavior is therefore fundamental to game designers. In this paper, we propose a dynamic model of consumer level-progression decisions to shed light on the underlying motivational drivers. We cast the individual play-or-quit decisions in a dynamic framework with forward-looking players and consumer learning about the evolution patterns of their operation efficiencies (defined as the average score earned per operation for passing a level). We develop a boundedly rational approach to model how individuals form predictions of their own operation efficiency and playing utility. This new approach allows researchers to flexibly capture players' over/unbiased/under estimation tendencies and risk averse/neutral/seeking preferences, two features that are particularly relevant when modeling the game-playing behavior. We develop an algorithm for estimating such dynamic model, and apply our model to level-progression data from individual players with one online game. We find that players in the sample tend to overestimate their operation efficiency, as their predicted values are significantly higher than the mean estimates inferred from their playing history with their completed levels. Furthermore, players are found risk seeking with moderate amount of uncertainty. We uncover two segments of players labeled as "Experiencers" vs. "Achievers": while the former tend to derive a higher utility from the playing process, the latter are more goal oriented and derive a higher benefit from completing the entire game. Two counterfactual simulations demonstrate that the proposed model can help adjust the uncertainty level and configure a more effective level-progression point schedule to better engage players and improve the game developer's revenue.
\end{abstract}

Keywords: Learning, Bounded Rationality, Prediction Bias, Risk Preference, Choice Model, Dynamic Structural Model, Online Gaming 


\section{Introduction}

The online game industry has enjoyed explosive growth since coming into being in the 1990's, forming a highly valuable segment of the digital economy. Playing games online has now become a major pastime for a constantly broadening demographic. As of 2011, Americans reported spending more time playing video games on casual websites than with consoles. The sales revenue from online gaming reached 8.3 billion dollars in US in 2012, and China saw a $34 \%$ increase in games revenue in the same year where online gaming made up 94\% of the pie (ESA Report 2013).

Many online games nowadays incorporate a system of level progression, in which users complete missions, earn points, and get promoted to the next level once their performance satisfies a certain criterion. For example, in the popular game Candy Crush Saga, players swipe candies to create sets of matching candies. Each level has a target score and this can be reached by accomplishing certain goals such as forcing items to fall to the bottom. A player will be promoted to the next level when she gets the target score. In another popular game Alpha 8: Airborne, players virtually select different cars and race in different locations. Players will then progress through levels of the game based on a point system.

A good understanding of user level progression is crucial for the success of online game developers. This is because level progression not only reflects user engagement, but also cultivates customer loyalty. From the firms' perspective, the more they can engage users through level progression, the more profitable they will be via selling virtual goods and displaying ads, the two primary revenue sources for game companies. Insights gained from the underlying motivational drivers of level progression can help game companies improve their level design. For example, game companies can adjust the uncertainty level by changing how they issue bonus points, or 
change the cutoff points associated with each level progression to better engage players and increase their revenue potential.

To better understand these issues, we specify and estimate a model of game-players' behavior as they progress through one online game. We consider a model in which a player decides to continue to play or quit the game at each level by maximizing the predicted utility accrued from the current period and the future. At each level, the player predicts her operation efficiency (defined as the average score earned per operation for passing a level), and weighs the utility benefits from continuing to play versus the costs and challenges of completing additional levels. These benefits and costs associated with game play change over levels, and the player also gets an extra utility after completing the entire game. Our premise is that the more levels the player completes, the more information she gains and learns about how her operation efficiency evolves. Before starting a new level, the player predicts her future operation efficiency and utility, and decides whether to continue to play or quit the game by maximizing the sum of the discounted utility from that point on.

Our model differs from other forward-looking learning models in the literature by allowing players' belief formation regarding their operation efficiency to be not fully rational, but rather incorporate features of bounded rationality to accommodate the quick split-second decisions which players must make in a computer game setting. Our empirical results further demonstrate that our model, with boundedly rational belief formation, explains the observed data substantially better than a standard model with beliefs formed via rational expectations. More importantly, our proposed boundedly rational approach is supported by psychological theories and captures two key features of user online gaming behavior. 
First, under bounded rationality, players' predictions of their future operation efficiency in the game can differ systematically from the expected values or the means based on past performance. For example, the self-serving bias (e.g. Folkes 1988), a well-established psychological theory, suggests that people tend to attribute successes to their own character or actions (i.e. internal attribution), but blame negative results to external factors (i.e. external attribution). Such self-serving bias can lead to overconfidence and overestimation of future performance (Libby and Rennekamp 2012). In this case, the player believes that the future event would unfold as a more positive outcome, and consequently her estimate of her future operation efficiency lies above her mean prediction based on her past performance. On the other hand, underestimation may also happen. For example, previous research finds that when people perceive a task with high difficulty, they may underestimate their ability (Kim et al. 2016). In that case, the player's predicted efficiency tends to be lower than her mean estimate based on her past performance.

Such systematic deviations between predicted and mean efficiency are especially relevant in the gaming context. This is because players may believe that they can partially influence the future outcomes. For instance, an overconfident player may readily attribute poor efficiency to playing mistakes which can be corrected in the future, leading to overestimation. Such systemic biases may be less relevant when consumers form expectations of the quality of a physical product. Imagining that when a consumer gets a low-quality product, she does not have much control over whether the same negative experience will repeat in the future; hence, her perception of the product quality in the future is likely to be objective, and not deviating systematically from the mean estimate inferred from her past experience. 
Systematic biases in beliefs have been widely documented in the literature. For example, evidence shows that people tend to overestimate their ability in preventing personal risks (Weinstein 1989), the level of control they have on an outcome (Langer 1975), and precision of their estimates (Oskamp 1965). Prior research finds that people are unrealistically optimistic in making predictions regarding their future goal pursuit (Buehler, Griffin, and Ross 1994, Zauberman and Lynch 2005), and therefore believe more goal-congruent activities will be accomplished in the future than in the past and overestimate future performance. Literature also documents that people with high self-esteem are more likely to overestimate the likelihood of positive outcomes (Brown, Collins and Schmidt 1988, Taylor and Brown 1988). On the other hand, there is evidence suggesting people's tendency to underestimate in some situations. For example, Billeter, Kalra and Loewenstein (2011) find that consumers tend to underestimate their skill learning ability after initially trying new products. However, our paper is among the first to incorporate these biases in beliefs into a structural dynamic choice model.

Second, under bounded rationality, players can have a concave utility function with respect to operation efficiency while showing risk seeking. However, as a general property, expected utility maximization involving a concave utility function leads to risk aversion (Bernoulli 1954). Although risk aversion is a sensible assumption used in modeling decision makers' risk attitudes in a wide range of situations, it may not be a universal behavioral tendency. A moderate amount of uncertainty can be beneficial to consumers in the gaming context, because players may enjoy some uncertainty since it allows new discovery and brings excitement. This is consistent with the sensation-seeking type of behavior as documented in the literature: individuals search for experiences and feelings that are varied, novel, complex and intense, and risk may be considered to add to the excitement of the activity (Zuckerman 1994). The literature has also shown that 
decision makers' risk seeking is associated with over confidence (e.g. Camerer and Lovallo 1999, Hirshleifer and Luo 2001). The findings from our preliminary data analysis show that while the play-or-quit decisions have an inverted-U relationship with the expected mean efficiency, players can be risk seeking. We specify the predicted utility as a function of the predicted efficiency in our boundedly rational framework, so that it allows us to flexibly capture the risk-averse, risk-neutral or risk-seeking behavior under a concave utility function.

We apply our proposed model to a panel dataset of consumer level progression with an online game. The game has multiple levels, and each level has a pre-specified required number of points for passing. For each player, we observe the level at which the user quit the game. For each completed level, we observe the number of operations the player performed. Our structural modeling approach allows us to identify two interesting segments of players: "Experiences" who derive a higher utility from the process of playing vs. "Achievers" who derive a higher benefit from completing the entire game. While both groups display overestimation of their operation efficiency, experiencers do so in a larger magnitude than achievers. These insights can help game companies more effectively design and market their products to players. Our two counterfactual analyses further dive deep into two design related issues. The first simulation illustrates the value of a reasonable amount of uncertainty (e.g. in the form of bonus points) in keeping consumers for playing more levels. The second simulation illustrates how our proposed model can be used to configure a more effective level-progression point schedule over game levels. The simulations can help game developers improve designs to better engage players and increase revenue.

With the proposed new model and managerial implications, our research contributes to a small yet growing body of marketing literature on user online gaming behavior. For example, Albuquerque and Nevskaya (2012) study player participation from an online computer game with 
frequent product updates of additional content and features. Nevskaya and Albuquerque (2019) propose a continuous-demand model to analyze consumer screen usage with an application to online gaming. Huang, Jasin, and Manchanda (2019) build a Hidden Markov Model (HMM) to capture the evolution of gamers' latent engagement states, and then use their findings to develop a matching algorithm to optimally pair up players to increase game-play. Our study is new in several aspects. First, while previous studies analyze consumer game playing activities, ours focuses on the player level-progression decision, a highly relevant metric to gaming companies. Second, we develop a new model to flexibly capture players' over/unbiased/under estimation tendencies and risk averse/neutral/seeking preferences. Third, our proposed model can be used to configure a more effective point schedule of cutoff points over game levels to improve user engagement, an important game design issue which has not be studied in the past.

The remainder of this paper is organized as follows. In section 2 , we introduce the background information of the empirical context and features of our data. In Section 3, we develop a dynamic structural model of consumer play-or-quit decision, by proposing a boundedly rational approach that allows researchers to flexibly capture the key behavioral tendencies in online gaming. In section 4, we discuss our findings and derive managerial implications via counterfactual simulations. Section 5 concludes the paper with implications for future research.

\section{Empirical Context and Data}

We have applied our model to an online game with 24 levels. It is a simple cooking game launched around 2011. In this game, a player takes on the role as a bakery owner. The player fulfills customers' requests/orders (or tasks) to earn points to advance to higher levels of the game. For example, if a customer orders a pound cake, the player needs to take the following steps: (1) Mix 
together the butter and cream cheese until they are light and fluffy; (2) Add salt, sugar, flour, milk, eggs and baking powder, and mix to combine; (3) Spoon the batter into the prepared loaf pan; and (4) bake for 90 minutes at 325F. Each step requires one successful operation, and in this case, the player needs at least 4 operations to complete the task. If the player makes mistakes in any step, the system will provide hints and allow the player to redo it, which means that the player will need more than four operations for this task. In other words, an operation can be defined as an action for completing an intermediary step, and multiple operations may be needed for completing a step if any of the operations are unsuccessful. Each level of the game consists of a number of similar tasks. At higher game levels, the tasks become more difficult, and the possible operations expand to selection of materials, tools, bakery time and temperature, decorations, actions, etc.

At the beginning of the game and during the process of playing, players are fully informed about the 24 levels and the incremental required passing points for each level $\left(G_{t}\right)$ where $t$ stands for game level. At each level, new tasks (e.g. cakes) are introduced, and these tasks are different but similar in terms of their difficulty level. As the game advances, the difficulty level of the tasks often increases. We get to observe operation quantity $\left(\mathrm{O}_{\mathrm{it}}\right)$ each player completed at each level (i for player and t for game level), and at which level the player quit the game. When reaching the required points for a level, the player will be promoted to the next level. With data on both operation quantity $\mathrm{O}_{i t}$ and incremental required passing points $\mathrm{G}_{t}$, we define operation efficiency $\left(\mathrm{A}_{\mathrm{it}}\right)$ as the ratio of the required points and the operation quantity incurred for passing a level, that is, $\mathrm{A}_{\mathrm{it}}=\mathrm{G}_{\mathrm{t}} / \mathrm{O}_{\mathrm{it}}$.

This game, while relatively simple, offers a clean and good fit to our single-agent dynamic optimization framework for a number of reasons. It is a single-player game without team or multiplayer aspects. Hence one player's play-or-quit decisions impose no externalities on other players' 
decisions. Moreover, there is no in-game spending as the gaming revenue derives completely from in-game advertising.

However, the study game retains the key features of a gaming context where a player makes play-or-quit decisions. Number of operations is not only a cost but also a venue to enjoy the playing, and players make play-or-quit decisions based on the predicted number of operations (or predicted operation efficiency given the deterministic relationship between operation quantity and operation efficiency). Previous research used latent variables to statistically model player engagement and how it affects user game-playing activities (Huang, Jasin, and Manchanda 2019). The advantage of our data is that we observe individual players' operation quantity/efficiency at each game level, which can be used to quantify user engagement with the game.

Although our empirical context may not capture every single feature of any game, we believe that our model can be adapted to other gaming contexts where additional covariates and decision variables can be incorporated. The relatively simple context also allows us to cleanly illustrate that players may over/under estimate their future operation efficiency and utility, and can be risk seeking with a concave utility function. These empirical analyses also enable us to carry out two important counterfactual analyses regarding how to vary the uncertainty level (e.g. through creating surprising bonus points) and configure a more effective level-progression point schedule to increase user engagement.

Table 1a reports the level-specific summary statistics on the incremental required passing point, the operation quantity, operation efficiency, and player attrition rate at each level from a random sample of 592 players who completed at least 3 levels of the game. We use data from levels 1-3 to construct the priors for initiating the player learning process, and data from levels 424 (a total of 21 levels) to estimate the proposed level-progression model. For easier interpretation 
of the data, we also present summary statistics on the same set of measures across different tiers of levels. ${ }^{1}$ In particular, we combine the first four levels, and for the remaining 20 levels, we group them into four tiers with 5 levels in each tier. To see clearly the variation of the data within the same game level, we also report the mean, standard deviation, minimum and maximum value of player operation quantity and operation efficiency in Table $1 \mathrm{~b}$ and Table $1 \mathrm{c}$ respectively.

$$
==\text { Insert Tables } 1 \mathrm{a}, 1 \mathrm{~b} \text { and } 1 \mathrm{c} \text { Here }==
$$

A close look at Table 1a reveals some key patterns. First, players' average operation quantity increases with game level, which may indicate that the game is getting more demanding and requires more operations to complete a level. Second, player operation efficiency shows a clear increasing trend. Note that operation efficiency measures the number of points earned per operation for passing a level, and it may reflect a player's ability as well as the game's difficulty. Since both player ability and game difficulty should progress step by step, we believe that an autoregressive model with one lag, AR1, is a reasonable model to capture the evolution of $\mathrm{A}_{\mathrm{it}}$ over game levels. Empirically, AR1 specification produces a good fit of players' observed efficiency data with an R-squared of 0.94 . Third, we observe that the average attrition rate is going down as players progress towards higher levels. This pattern may suggest that players are forward looking, and as they level up, they are more likely to stay as they care about future gains. We also notice that the attrition rate has a sharp decline in the last five levels (i.e. dropping from 0.15 to 0.1 ). This may suggest some additional utility gain from finishing the entire game, and furthermore, due to discounting of future utility, this additional utility affects the play-or-quit decisions more strongly towards the end of the game.

\footnotetext{
${ }^{1}$ This grouping is made just for the ease of observing data patterns, and we do not apply such level grouping in model calibration.
} 
To more clearly see the operation efficiency trajectories for the same group of players without self-selection, we have plotted the average operation efficiencies for four groups. Group6 represents the group of players who completed (and only completed) the first 6 levels, and similarly we define Group12, Group18 and Group24. We have two observations. First, in general, the operation efficiencies increase as players advance to higher levels, and this applies to all groups of players. Second, players in Group24 who completed the whole game have relative lower operation efficiency. A possible explanation is as follows. Given the same required passing points, low efficiency means more operations. We know that players have an overall net positive utility from an operation. Therefor players with low efficiency are more likely to continue to play the game.

$$
==\text { Insert Figure } 1 \text { Here }==
$$

A player makes a play-or-quit decision at each level of the game, and our premise is that the playing utility is affected by operation quantity or efficiency, because there is a deterministic relationship between the two $\left(\mathrm{A}_{\mathrm{it}}=\mathrm{G}_{\mathrm{t}} / \mathrm{O}_{\mathrm{it}}\right)$ and the incremental required passing points $\mathrm{G}_{\mathrm{t}}$ are given and fully observed by players at the beginning of the game. When the player makes a play-or-quit decision, the operation efficiency is not fully observed, and may require the player to make a prediction based on past experience. In order to draw insights from data to guide us in model development, we ran a preliminary data analysis to show how the play-or-quit decisions are related to the perceived mean and variance of the operation efficiency, by taking three steps: 1) Run the AR1 regression on the observed operation efficiency data and estimated the variance of the error term; 2) Use the Bayesian-updating formulation to construct the mean and variance of the efficiency at each level of the game; 3) Run a logistic regression on the play-or-quit decision (1 for play and 0 for quit) at each level for each consumer, using the following covariates: mean efficiency ("Mean"), square term of the mean efficiency ("MeanSquare"), the variance of the 
efficiency ("Variance"), and the game level dummies. All the covariates are rescaled by dividing them by 10 .

We find that the coefficient of "Mean" is significantly positive (Estimate $=2.824$, P-value $=0.030$ ), and "MeanSquare" has a significantly negative coefficient (Estimate $=-0.396$, P-value $=0.005)$, suggesting that players' continuation decision has an inverted-U relationship with the expected efficiency. Most interestingly, we find that the perceived variance of the efficiency has a positive sign $($ Estimate $=0.798, \mathrm{P}$-value $=0.032)$, suggesting the possibility of risk seeking. There are two possible explanations for these data patterns: 1) Players make a forward-looking decision rather than myopic decision, because they are willing to take the current risk to reduce the future uncertainty to get a higher overall utility; 2) Players are likely over confident and overestimating their operation efficiency, casting doubts on the rational expectations assumption.

Given the reported data patterns, we assume a utility function with diminishing return from the expected efficiency, and we propose a boundedly rational approach to flexibly capture players' over/unbiased/under estimation tendencies and risk averse/neutral/seeking preferences under such concave utility function. We also allow for forward looking when players make the play-or-quit decisions over the game levels.

\section{Model Development}

Our general modeling context is online gaming with multiple levels indexed by t. Completion of level $t$ requires earning a pre-specified number $\mathrm{G}_{\mathrm{t}}$ of points. For a player indexed by $\mathrm{i}(\mathrm{i}=1, \ldots$, I), we observe the level at which she quits denoted by $\mathrm{T}_{\mathrm{i}}$. For each completed level, we observe the number of operations $\left(\mathrm{O}_{\mathrm{it}}\right)$ which player i incurred in finishing the level; since players with a higher operation efficiency will manage to earn the required points with a fewer number of 
operations, we define player i's operation efficiency as the ratio of required points to operations: $A_{i t}=G_{t} / O_{i t}$. Each player makes a play-or-quit decision at the beginning of each level $t$, which we denote as $\mathrm{D}_{\mathrm{it}}$.

\subsection{Per-Period Utility}

A player's per-period utility of finishing level $t$ is:

$U_{i t}=\beta_{i t} \ln \left(1+O_{i t}\right)-\kappa_{i t} O_{i t}+S O A_{i t}+\eta_{i t}$

The first term, $\beta_{i t} \ln \left(1+O_{i t}\right)$, captures the player's gain or enjoyment from playing at level $\mathrm{t}$, and we constrain $\beta_{i t}$ to be positive. Diminishing returns from operations are imposed via the logarithmic functional form. The second term, $\kappa_{i t} O_{i t}$, captures the cost of time and energy incurred from those operations when playing level t, and we constrain the coefficient $\kappa_{i t}$ to be positive to reflect operations to be a cost. The third term, $S O A_{i t}$, captures the sense of accomplishment or satisfaction from completing level t. The fourth term, $\eta_{i t}$, is an idiosyncratic individual and level specific error term, capturing all other factors that affect a player's utility unobserved by researchers, and we assume it follows a standard normal distribution: $\eta_{i t} i i d \sim N(0,1)$. We can mathematically show that equation 1 is a concave utility function with respect to $\mathrm{O}_{\text {it }}$ and with respect to $A_{i t}$ given the deterministic relationship of $A_{i t}=G_{t} / O_{i t}$, when $\beta_{i t}>0$ and $\kappa_{i t}>0$.

We allow the parameters in the utility function to be heterogeneous across consumers and game levels, taking the following specifications,

$\beta_{i t}=\exp \left(\gamma_{i 0}+\gamma_{i 1} t\right)$

$\kappa_{i t}=\exp \left(\omega_{i 0}+\omega_{i 1} t\right)$

$S O A_{i t}=\tau_{i 0}+\tau_{i 1} t$

These parameterizations lead to interesting predictions of player preferences over the gaming process. For instance, $\gamma_{i 1}$ captures how the gain from an operation changes as the player progresses 
over game levels. If $\gamma_{i 1}>0$, the player enjoys game more as she is advancing through the levels. If $\gamma_{i 1}<0$, the player becomes less engaged and gradually losing interest as she continues. We also expect the marginal cost of operation $\kappa_{i t}$ to decrease with game levels as the player is accumulating more experience. Finally, we postulate that sense of accomplishment $S O A_{i t}$ may vary with respect to game levels t.

At the beginning of level $t$, the player decides whether to continue or quit the game. We fix the utility from the outside option to zero: ${ }^{2}$

$O U_{i t}=0$

In our setting, the player makes a play-or-quit decision without full knowledge of the number of operations she needs to pass the next level. Hence, she needs to form a prediction on the operation quantity. We discuss this next.

\subsection{Learning about Operation Efficiency}

Operation efficiency is unlikely to be constant, because players may learn from the evolution of the operation efficiency and game designs change over levels. Therefore, unlike most papers in the empirical learning literature in which decisions makers learn a constant (time-invariant) element, such as product match-quality, we model how gamers learn about the changes in, or evolution of, their time-varying operation efficiency. ${ }^{3}$ We model log efficiency as an evolving AR1 process:

$\ln A_{i t}=\alpha_{0 i}+\alpha_{1 i} \ln A_{i, t-1}+\varepsilon_{i t}$

where $\varepsilon_{i t}$ iid $\sim N\left(0, \sigma_{i}^{2}\right) . \alpha_{0 i}$ and $\alpha_{1 i}$ are coefficients that determine the evolution of operation efficiency over levels. We make those coefficients individual-specific to allow players to have

\footnotetext{
${ }^{2}$ In the discrete choice framework, the value of the outside option cannot be separately identified from $\tau_{i 0}$.

${ }^{3}$ This is related to the literature on learning-by-doing (e.g. Arrow 1962, and Chan, Li and Pierce 2014).
} 
different evolution patterns due to different learning abilities. Since the AR1 process implies the mean $\log$ efficiency at a steady state to be $\alpha_{0 i} /\left(1-\alpha_{1 i}\right), \alpha_{0 i}$ can be interpreted as the baseline and $\alpha_{1 i}$ as the persistence.

Following Chylinski, Roberts and Hardie (2012), we assume that each player is uncertain about the evolutionary pattern of her operation efficiency, as measured by the parameters $\alpha_{0 i}$ and $\alpha_{1 i}$, and learns about them from her past performance. We define

$\tilde{\alpha}_{i}=\left[\begin{array}{c}\alpha_{0 i} \\ \alpha_{1 i}\end{array}\right]$,

$Y_{i t}=\ln A_{i t}$,

$X_{i t}=\left[\begin{array}{ll}1 & \ln A_{i, t-1}\end{array}\right]$.

Given the normality assumptions, at the beginning of each level $t$, each player's current beliefs about $\tilde{\alpha}_{i}$ take the form of a bivariate normal distribution with mean $\mu_{i t}$ and variance-covariance $\Sigma_{i t}$ which vary across levels:

$\tilde{\alpha}_{i} \mid I_{i t} \sim M V N\left(\mu_{i t}, \Sigma_{i t}\right)$

where $I_{i t}$ is the information set at the beginning of $\mathrm{t}$. After finishing level $\mathrm{t}$, the player observes her realized $\mathrm{A}_{\mathrm{it}}=\mathrm{G}_{\mathrm{t}} / \mathrm{O}_{\mathrm{it}}$. She then updates her evaluation on $\tilde{\alpha}_{i}$ at the beginning of $\mathrm{t}+1$ in a Bayesian fashion:

$\tilde{\alpha}_{i} \mid I_{i, t+1} \sim M V N\left(\mu_{i, t+1}, \Sigma_{i, t+1}\right)$

where

$\mu_{i, t+1}=\Sigma_{i, t+1}\left(\Sigma_{i t}^{-1} \mu_{i t}+X_{i t}{ }^{\prime} Y_{i t} / \sigma_{i}^{2}\right)$

$\Sigma_{i, t+1}^{-1}=\Sigma_{i t}^{-1}+X_{i, t}{ }^{\prime} X_{i, t} / \sigma_{i}^{2}$.

Given the information set $I_{i t}$, according to equations 4 and 6 , the player's belief on her efficiency should follow:

$\ln A_{i t} \mid I_{i t} \sim N\left(m_{i t}, \Delta_{i t}^{2}\right)$ 
where $m_{i t}=X_{i t} \mu_{i t}$ and $\Delta_{i t}^{2}=X_{i t} \Sigma_{i t} X_{i t}^{\prime}+\sigma_{i}^{2}$.

\subsection{Utility Specifications under Uncertainty}

The player faces uncertainty about her efficiency as well as the required operation quantity for completing the next level. Given the uncertainty on $\ln \mathrm{A}_{\mathrm{i}}$, how do players predict their playing utility in equation 1? We consider two alternative approaches in this paper. First, we assume players have rational expectations which they use to predict their playing utility. Second, we consider a novel boundedly rational approach to flexibly capture players' over/under estimation behavior and risk preferences, which avoids the strong and restrictive assumptions of rational expectations. We describe each alternative in turn.

\subsubsection{Rational Expectation Approach}

In the expectation-based approach, the player forecasts her efficiency, and the associated operation quantity and utility. Specifically,

$E U_{i t}=\beta_{i t} E \ln \left(1+O_{i t}\right)-\kappa_{i t} E\left(O_{i t}\right)+S O A_{i t}+\eta_{i t}$

The player forms an expectation on her playing utility based on the number of operations needed for promoting to the next level. Since operation quantity is uniquely determined by the incremental required passing points $G_{t}$ and $\log$ efficiency $\ln A_{\text {it }}$ (see equation 4), calculating expected utility with respect to operation quantity is equivalent to calculating expected utility with respect to the $\log$ efficiency, given the uncertainty on the player's efficiency. ${ }^{4}$

When $\mathrm{O}_{\mathrm{it}}$ is reasonably large, say greater than 10 , we have $\ln \left(1+O_{i t}\right) \approx \ln \left(O_{i t}\right)$. Equation 9 can be then approximated as,

$$
E U_{i t} \approx \beta_{i t} E \ln \left(O_{i t}\right)-\kappa_{i t} E\left(O_{i t}\right)+S O A_{i t}+\eta_{i t}
$$

\footnotetext{
${ }^{4}$ Note that error term $\eta_{i t}$ is observed by player although not by the researcher, and therefore the player does not need to form expectation on the measurement error $\eta_{i t}$.
} 


$$
\begin{aligned}
& =\beta_{i t} E \ln \left(G_{i t} / A_{i t}\right)-\kappa_{i t} E\left(G_{i t} / A_{i t}\right)+S O A_{i t}+\eta_{i t} \\
& =\beta_{i t}\left(\ln G_{t}-E \ln A_{i t}\right)-\kappa_{i t} G_{t} E e^{-\ln A_{i t}}+S O A_{i t}+\eta_{i t}
\end{aligned}
$$

Then using the property of the moment generating function (MGF) of lognormal distribution, we can derive the player's expectation-based predicted utility (EU) at the beginning of level t as,

$E U_{i t} \approx \beta_{i t}\left(\ln G_{t}-m_{i t}\right)-\kappa_{i t} G_{t} e^{-m_{i t}+\frac{\Delta_{i t}^{2}}{2}}+S O A_{i t}+\eta_{i t}$

where $m_{i t}$ is the player's belief on her mean log efficiency, and $\Delta_{i t}^{2}$ is the variance or uncertainty of the belief, according to equation 8. Players are assumed to evaluate the complicated utility function by integrating out the uncertainty. Moreover, equation $10 \mathrm{~b}$ implies that $E U_{i t}$ decreases as uncertainty increases since $\kappa_{i t}>0$, suggesting that players are risk averse.

While risk aversion is a sensible one, and has been adopted in other empirical studies of dynamic choice under uncertainty (Erdem and Keane 1996, Chintagunta, Jiang and Jin 2009, Ching and Ishihara 2012, and Zhao et al. 2013), it may be less appropriate to impose in our online gaming context, where players may be risk seeking as they enjoy a moderate amount of uncertainty for new discovery and extra excitement (as demonstrated in our preliminary data analysis). In the next section, we extend the model to allow for a variety of risk preferences, as well as bounded rational beliefs and decision-making.

\subsubsection{Boundedly Rational Approach}

We now consider an alternative specification on the predicted utility which extends the previous specification to allow for bounded rationality in playing behavior. This specification accommodates a variety of risk preferences, and at the same time allows players' beliefs about their efficiencies to be systematically higher or lower than would be warranted by strict Bayesian learning. 
Based on the utility function in equation 1, we specify the predicted utility (PU) as a function of the predicted operation quantity $\mathrm{PO}_{i t}$ where $\mathrm{PO}_{i t}=\mathrm{G}_{t} / \mathrm{PA}_{\mathrm{it}}, \mathrm{PA}_{\mathrm{it}}$ is the predicted efficiency, and $\mathrm{G}_{\mathrm{t}}$ is the incremental points required for completing level $\mathrm{t}$, which is fixed. We then specify the predicted efficiency $\mathrm{PA}_{\mathrm{it}}$ as $e^{m_{i t}+l_{i} \Delta_{i t}}$. This expression, with the extra parameter $l_{i}$, captures several types of biases in parsimonious fashion. According to equation $8, \ln \mathrm{A}_{\mathrm{it}}$ is distributed normal with mean $m_{i t}$ and variance $\Delta_{i t}^{2}$. Hence $l_{i}$ captures the player i's attitude toward uncertainty: $l_{i}>0$ suggests that the player overestimates her $\log$ efficiency $\left(\ln \mathrm{A}_{\mathrm{it}}\right)$ to be above the mean $\left(m_{i t}\right)$. On the other hand, $l_{i}<0$ may indicate that the player predicts her log efficiency $\left(\ln \mathrm{A}_{i t}\right)$ to be below the mean $\left(m_{i t}\right)$. In the knife-edge case of $l_{i}=0$, the player is neither systematically over or under estimating since she uses her mean predication based on her past performance to forecast her future efficiency. Our boundedly rational approach thus specifies the player predicted utility as,

$$
\begin{aligned}
P U_{i t} & =\beta_{i t} \ln \left(1+P O_{i t}\right)-\kappa_{i t} P O_{i t}+S O A_{i t}+\eta_{i t} \\
& =\beta_{i t} \ln \left(1+G_{t} / e^{m_{i t}+l_{i} \Delta_{i t}}\right)-\kappa_{i t} G_{t} / e^{m_{i t}+l_{i} \Delta_{i t}}+S O A_{i t}+\eta_{i t}
\end{aligned}
$$

Compared to equation $10 \mathrm{~b}$, we see that the standard deviation term $\Delta_{i t}$ now enters the predicted utility in equation 11 in the first two components: the predicted gains and the predicted losses. This accommodates a variety of risk preferences. When $l_{i}>0$, the first term decreases in uncertainty while the second term increases in uncertainty. Hence equation 11 is flexible in capturing all three types of risk preferences depending on the magnitude of model estimates: risk aversion, risk neutral, and risk seeking. The same logic applies to the case of $l_{i}<0$. This marks a key difference between the boundedly rational approach and the rational expectations approach.

To further verify the flexibility of the model on players' risk attitudes, we can derive the first-order condition of the predicted utility with respect to the uncertainty $\Delta_{i t}$ as follows, 
$\frac{\partial P U_{i t}}{\partial \Delta_{i t}}=-l_{i}\left(\frac{\beta_{i t}}{1+G_{t} / e^{m_{i t}+l_{i} \Delta_{i t}}}-\kappa_{i t}\right) G_{t} / e^{m_{i t}+l_{i} \Delta_{i t}}$

This equation clearly indicates that individual players' risk preference can be positive, zero or negative, and it is affected by $l_{i}$ (capturing the player's attitude toward uncertainty) and $\Delta_{i t}$ (capturing the magnitude of the uncertainty on efficiency) under the boundedly rational approach. For example, when $l_{i}>0$ and $\Delta_{i t}$ is relatively small, $\frac{\beta_{i t}}{1+G_{t} / e^{m_{i t}+l_{i} \Delta_{i t}}}-\kappa_{i t}$ is likely to be smaller than zero and in turn $\frac{\partial P U_{i t}}{\partial \Delta_{i t}}$ will be larger than zero (i.e. risk seeking). However, if $\Delta_{i t}$ passes a threshold value, $\frac{\beta_{i t}}{1+G_{t} / e^{m_{i t}+l_{i} \Delta_{i t}}}-\kappa_{i t}$ will turn positive, suggesting a negative value for the derivative (i.e. risk aversion).

\subsection{Players' Dynamic Optimization Problem}

Since exit decisions are irreversible, a player's decision to quit the game at level t prevents her from enjoying higher levels of the game. Hence, we assume players to be forward looking, that is, to make a play-or-quit decision at each level of the game to maximize the sum of current period utility and discounted future utility. The Forward-looking assumption has been adopted to model consumer decisions in a wide variety of marketing contexts (Erdem and Keane 1996, Hartmann 2006, Albuquerque and Nevskaya 2012, and Lee, Kumar and Gupta 2014). Since a game has a finite number of levels, we consider a finite-horizon dynamic problem.

For each level $\mathrm{t}$, the timing is as follows: 1) The player observes mean $\mu_{i t}$ and variance $\Sigma_{i t}$ of the two coefficients $\alpha$ that determine her efficiency evolution and the log-efficiency realized at level t-1 $\ln A_{i, t-1}$. 2) The player also observes $\eta_{i t}$, the stochastic error term in the utility, which is unobservable to the researcher. 3) The player makes a play-or-quit decision based on the information set in the previous two steps. 4) If she chooses to quit at level $t$, she does not make any decisions onwards and her learning process discontinues; Otherwise, she finishes level $\mathrm{t}$ and 
observes her realized efficiency at level $\mathrm{t}\left(A_{i t}\right)$. 5) The player updates her belief on the two coefficients that govern how her efficiency evolves over levels after completing level t. Posterior beliefs are formed after competing level t and will be used as the player's prior at the beginning of level $\mathrm{t}+1$. 6) We assume that if a player finishes all the $\mathrm{T}$ levels in the game, she will get an extra utility $C_{i}$, to reflect game players' additional gain or loss due to finishing the entire game. It reflects a widely observed psychological phenomena: the need for closure (Kruglanski and Webster 1996).We expect the parameter $C_{i}$ to be positive, but will estimate this parameter freely and let data informs us of its sign and magnitude. The sharp decline of attribution in the last five levels as shown in Table 1a is consistent with the specification of $\mathrm{C}_{\mathrm{i}}$, and we have elaborated the rationale in Section 2.

At the beginning of each level $t$, the player makes the play-or-quit decision by maximizing the sum of current period utility and discounted future utility, i.e. the present discounted value (PDV). Since PDV involves components the player is uncertain about (i.e. current and future efficiency and future random errors), she needs to form predictions on them. As a result, calculation of the PDV has to be based on player predictions on these uncertain components. At the beginning of level $t$, the player's decision problem can be written as,

$\underset{D_{i t}, \ldots, D_{i T}}{\operatorname{Max}} \sum_{k=t}^{T} \delta^{k-t} I\left(D_{i k}=1\right) \times \operatorname{Predicted}\left(U_{i k} \mid S_{i k}, \eta_{i t}\right)+C_{i} I\left(D_{i T}=1\right)$

where we use "Predicted" to denote predicted utility under observed state variables $S_{i k}$ and latent state variables $\eta_{i t}$. To summarize, we have adopted the following notations in our dynamic model formulation.

$M_{i t} \quad$ Deterministic part of the expected (or predicted) period utility

$C_{i} \quad$ Extra utility if player finishes all levels.

$S_{i t} \quad$ Observed state variables. 
$\delta \quad$ Discount factor

$V_{i t}(\cdot) \quad$ Value function for player $\mathrm{i}$ at level $\mathrm{t}$, a function of all state variables.

$V_{i t}^{*}(\cdot)$ Ex-ante Value function for player $\mathrm{i}$ at level $\mathrm{t}$, a function of observed state variables.

$D_{i t} \quad$ Player $i$ 's decision at level t. If player i quit the game at task $\mathrm{t}, D_{i t}=0$; otherwise $D_{i t}=1$.

\subsubsection{State Variables and Transition Probability}

Let $\left\{S_{i t}, \eta_{i t}\right\}$ denote the set of state variables for player $\mathrm{i}$ at the beginning of level $\mathrm{t}$, where $S_{i t}=$ $\left\{\mu_{i t}, \Sigma_{i t}, \ln A_{i, t-1}\right\}$ is the set of observable state variables and $\eta_{i t}$ is the unobservable state variable from the researcher's perspective. Transition rules of $S_{i t}=\left\{\mu_{i t}, \Sigma_{i t}, \ln A_{i, t-1}\right\}$ to $S_{i, t+1}=$ $\left\{\mu_{i, t+1}, \Sigma_{i, t+1}, \ln A_{i t}\right\}$ have been defined in equations $7 \mathrm{~b}, 7 \mathrm{c}$ and 8 . Note that, given $S_{i t}$, the $\mu_{i, t+1}\left(\Sigma_{i, t+1}\right)$ is determined by $\ln A_{i t}$ according to equation $7 \mathrm{~b}$ (equation $7 \mathrm{c}$ ). Therefore, we have $P\left(S_{i, t+1} \mid S_{i t}\right)$

$=P\left(\mu_{i, t+1}, \Sigma_{i, t+1} \mid \ln A_{i t}, S_{i t}\right) P\left(\ln A_{i t} \mid S_{i t}\right)$

$=P\left(\mu_{i, t+1}, \Sigma_{i, t+1} \mid \ln A_{i t}, S_{i t}\right) \varphi\left(\frac{\ln A_{i t}-X_{i t} \mu_{i t}}{\sqrt{X_{i t} \Sigma_{i t} X_{i t}^{\prime}+\sigma_{i}^{2}}}\right)$

$= \begin{cases}\varphi\left(\frac{\ln A_{i t}-X_{i t} \mu_{i t}}{\sqrt{X_{i t} \Sigma_{i t} X_{i t}^{\prime}+\sigma_{i}^{2}}}\right) & \text { if } \mu_{i, t+1}=\Sigma_{i, t+1}\left(\Sigma_{i t}^{-1} \mu_{i t}+\frac{X_{i t}^{\prime} Y_{i t}}{\sigma_{i}^{2}}\right), \Sigma_{i, t+1}^{-1}=\Sigma_{i t}^{-1}+\frac{X_{i t}^{\prime} X_{i t}}{\sigma_{i}^{2}} \\ 0 & \text { otherwise }\end{cases}$

where $\varphi$ is the probability density function of the standard normal distribution.

The unobserved state variables $\eta_{i t}$ are the error terms in the period utility function. We assume that they are i.i.d. following the standard normal distribution, and orthogonal to other covariates and random variables in the model. Therefore, this specification satisfies the conditional independence requirement for solving the DP problem (Rust 1988).

\subsubsection{DP under the Rational Expectation Approach}


We now derive the solution of the DP problem under the rational expectations approach. By Bellman's principle of optimality, the value function can be obtained using the recursion. Define $V_{i, T+1}=C_{i}$, which indicates that if player finishes all levels, she can get extra utility $C_{i}$. Then we have value function at $\mathrm{t}$ for $\mathrm{t}=1, \ldots, \mathrm{T}$ as,

$$
\begin{aligned}
V_{i t}\left(S_{i t}, \eta_{i t}\right)= & \max \left\{E_{A_{i t}}\left(U_{i t} \mid S_{i t}, \eta_{i t}\right)+\delta E_{S_{i, t+1}, \eta_{i, t+1}} V_{i, t+1}\left(S_{i, t+1}, \eta_{i, t+1} \mid S_{i t}, \eta_{i t}\right), \quad 0\right\} \\
& =\max \left\{M_{i t}+\eta_{i t}+\delta E_{A_{i t}} E_{\eta_{i, t+1}} V_{i, t+1}\left(S_{i, t+1}, \eta_{i, t+1} \mid S_{i t}, \eta_{i t}\right), \quad 0\right\}
\end{aligned}
$$

where $M_{i t}=\beta_{i t} E \ln \left(1+O_{i t}\right)-\kappa_{i t} G_{t} e^{-X_{i t} \mu_{i t}+\frac{1}{2}\left(X_{i t} \Sigma_{i t} X_{i t}^{\prime}+\sigma_{i}^{2}\right)}+S O A_{i t}$.

To reduce the dimension of the state space and simplify solving DP, we define the ex-ante value function, as follows:

$V_{i t}^{*}\left(S_{i t}\right)=\int V_{i t}\left(S_{i t}, \eta_{i t}\right) d F\left(\eta_{i t}\right)=E_{\eta_{i t}} V_{i t}\left(S_{i t}, \eta_{i t}\right)$

$\mathrm{V}_{\mathrm{it}}^{*}\left(\mathrm{~S}_{\mathrm{it}}\right)$ is player i's value of being in state $S_{i t}$, after integrating out $\eta_{i t}$. Applying the expected value of a truncated normal distribution, we have

$$
\begin{aligned}
V_{i t}^{*}\left(S_{i t}\right)= & E_{\eta_{i t}} \max \left\{M_{i t}+\eta_{i t}+\delta E_{A_{i t}} E_{\eta_{i, t+1}} V_{i, t+1}\left(S_{i, t+1}, \eta_{i, t+1} \mid S_{i t}, \eta_{i t}\right), \quad 0\right\} \\
= & \Phi\left(M_{i t}+\delta E_{A_{i t}} V_{i, t+1}^{\prime}\left(S_{i, t+1} \mid S_{i t}\right)\right) \times\left(M_{i t}+\delta E_{A_{i t}} V_{i, t+1}^{\prime}\left(S_{i, t+1} \mid S_{i t}\right)\right) \\
& +\phi\left(M_{i t}+\delta E_{A_{i t}} V_{i, t+1}^{\prime}\left(S_{i, t+1} \mid S_{i t}\right)\right)
\end{aligned}
$$

Then we have the choice-specific value function as

$$
v\left(S_{i t}, \eta_{i t}, D_{i t}\right)=\left\{\begin{array}{lr}
M_{i t}+\eta_{i t}+\delta E_{A_{i t}} V_{i, t+1}^{*}\left(S_{i, t+1} \mid S_{i t}\right) & \text { if } D_{i t}=1 \\
0 & \text { if } D_{i t}=0
\end{array}\right.
$$

Therefore, the probability of continuing to play is

$$
P\left(D_{i t}=1 \mid S_{i t}\right)=\Phi\left(M_{i t}+\delta E_{A_{i t}} V_{i, t+1}^{*}\left(S_{i, t+1} \mid S_{i t}\right)\right)
$$

\subsubsection{DP under the Boundedly Rational Approach}


We now derive the solution of the DP problem under our boundedly rational specification as explained in section 3.3.2. In this specification, the value function will be obtained following the standard formula, but conditional on $\ln A_{i t}=m_{i t}+l_{i} \Delta_{i t}$. The value function is now,

$$
\begin{aligned}
& V_{i t}\left(S_{i t}, \eta_{i t}\right)= \\
& \max \left\{M_{i t}+\eta_{i t}+\delta E_{\eta_{i, t+1}} V_{i, t+1}\left(S_{i, t+1}, \eta_{i, t+1} \mid S_{i t}, \eta_{i t}, \ln A_{i t}=m_{i t}+l_{i} \Delta_{i t}\right), \quad 0\right\}
\end{aligned}
$$

where $M_{i t}=\beta_{i t} \ln \left(1+G_{t} / e^{m_{i t}+l_{i} \Delta_{i t}}\right)-\kappa_{i t} G_{t} / e^{m_{i t}+l_{i} \Delta_{i t}}+S O A_{i t}$ is the deterministic part of utility.

Similarly, we can derive the value function based only on observed state variables,

$$
\begin{aligned}
& V_{i t}^{*}\left(S_{i t}\right)=\int V_{i t}\left(S_{i t}, \eta_{i t}\right) d F\left(\eta_{i t}\right)=E_{\eta_{i t}} V_{i t}\left(S_{i t}, \eta_{i t}\right) \\
& =E_{\eta_{i t}} \max \left\{E\left(U_{i t} \mid S_{i t}, \ln A_{i t}=m_{i t}+l_{i} \Delta_{i t}\right)+\delta V_{i, t+1}^{*}\left(S_{i, t+1} \mid S_{i t}, \ln A_{i t}=m_{i t}+l_{i} \Delta_{i t}\right), \quad 0\right\} \\
& =\Phi\left(M_{i t}+\delta V_{i, t+1}^{*}\left(S_{i, t+1} \mid S_{i t}, \ln A_{i t}=m_{i t}+l_{i} \Delta_{i t}\right)\right) \times\left(M_{i t}+\delta V_{i, t+1}^{*}\left(S_{i, t+1} \mid S_{i t}, \ln A_{i t}=\right.\right. \\
& \left.\left.\quad m_{i t}+l_{i} \Delta_{i t}\right)\right)+\phi\left(M_{i t}+\delta V_{i, t+1}^{*}\left(S_{i, t+1} \mid S_{i t}, \ln A_{i t}=m_{i t}+l_{i} \Delta_{i t}\right)\right) .
\end{aligned}
$$

Then we have the choice-specific value function is

$$
v\left(S_{i t}, \eta_{i t}, D_{i t}\right)=\left\{\begin{array}{lr}
M_{i t}+\eta_{i t}+\delta V_{i, t+1}^{*}\left(S_{i, t+1} \mid S_{i t}, \ln A_{i t}=m_{i t}+l_{i} \Delta_{i t}\right) & \text { if } D_{i k}=1 \\
0 & \text { if } D_{i k}=0
\end{array}\right.
$$

Therefore, the probability of playing is

$$
P\left(D_{i t}=1 \mid S_{i t}\right)=\Phi\left(M_{i t}+\delta V_{i, t+1}^{*}\left(S_{i, t+1} \mid S_{i t}, \ln A_{i t}=m_{i t}+l_{i} \Delta_{i t}\right)\right)
$$

\section{Empirical Application}

\subsection{Empirical Identification}

Several notes on the empirical identification of the model parameters are in order. First, unlike in other learning contexts where experience signals are unobserved to researchers, we directly 
observe learning signals which are players' realized efficiency after completing a level. Observing signals can greatly improve the identification of learning models (Ching, Erdem and Keane 2013). As such, equation 4 is a simple regression model where both dependent and independent variables are observed and vary across different levels. Furthermore, in order to strengthen the empirical identification, we have followed the learning model literature by estimating the initial conditions (i.e. mean and variance of $\alpha$ ) using her realized efficiency during the first three levels and equation 4. Given the initial conditions and observed signals, the mean and variance of a player's operation efficiency at each level are therefore uniquely determined following equation 8 . This is crucial because a player's expected utility at each level is a function of the mean and variance of her efficiency as shown in equation 11.

Second, $\beta$ and $\kappa$ can be loosely interpreted as the gain and cost of operations, and they can be empirically identified for three reasons. First, players do not quit at the same level, and the distribution of quitting agents across levels identifies those parameters across segments. Second, gain and cost are modeled with different functional forms and vary over levels. Third, expected operation quantities are empirically identified given their deterministic relationship with expected operation efficiency which is shown to be empirically identifiable given the previous argument. Our preliminary data analysis suggests that operation efficiency and utility follow an inverted $\mathrm{U}$ relationship. This suggests that, given a fixed required point $\mathrm{G}$ and the relationship of $\mathrm{O}=\mathrm{G} / \mathrm{A}$, a player's utility reaches the maximum value under a moderate operation quantity $\mathrm{O}$. With sufficient data on playing at each level, we can empirically infer the maximum utility (due to operation quantity) and the optimal operation quantity. Note that, with the functional form specified in our model, we have the optimal operation quantity as $\beta / \kappa-1$ and the maximum utility as $\beta \ln (\beta / \kappa)$ $\kappa(\beta / \kappa-1)$, that is, we have two equations (or two pieces of information) to identify two parameters. 
Therefore, we can empirically identify $\beta$ and $\kappa$ for each game level. The corresponding parameters $\gamma_{0}, \gamma_{1}, \omega_{0}$, and $\omega_{1}$ can be then identified through the co-variation between $\beta$ and $t$ and the co-variation between $\kappa$ and $\mathrm{t}$.

Third, $\mathrm{C}$ is the extra utility gained by a player who completes the whole game. It captures a widely observed psychological phenomenon: the need for closure. Since we adopt the dynamic programming specification to capture player's forward looking behavior, C will influence a player's utility at all levels rather than just at the last level, and the impact of $\mathrm{C}$ decreases exponentially as the focal level is further away from the last level of the game. As shown from Table 1, the attrition rate declines sharply in the last five levels (i.e. dropping from 0.15 to 0.1 ). This may suggest some additional utility gain from finishing the game. Furthermore, due to discounting of future utility, this additional utility affects the play-or-quit decisions more strongly towards the end of the game. The sharp decline of attrition in the last five levels is consistent with the specification of $\mathrm{C}$ and the magnitude of this decline helps us empirically identify $\mathrm{C}$.

Finally, the other important parameter $l_{i}$ which indicates the direction and magnitude of the expectation bias, is empirically identifiable because, as shown in equation 12, players' risk preferences (i.e. the effect of the variance of the operation efficiency on the expected playing utility) are affected by $l$. According to our preliminary analysis, there exist significant risk seeking for many players, and this data pattern will help us identify $l$ empirically.

\subsection{Model Estimation}

We now describe the model estimation procedure. We fix the discount factor $\delta$ to be 0.9 . We also tried other values such as 0.85 and 0.95 , and did not find any systematic changes of the results. Let $T_{i}$ be the last level completed by player i and $\Theta_{i}=\left\{\gamma_{0 i}, \gamma_{1 i}, \omega_{0 i}, \omega_{1 i}, \tau_{0 i}, \tau_{1 i}, C_{i}, l_{i}, \tilde{\alpha}_{i}, \sigma_{i}^{2}\right\}$ be the set of player i specific parameters. As in Rust (1988), the likelihood function in our dynamic 
optimization model consists of two components: the choice probabilities and probabilities of actual observed state evolution (rather than agents' believed state evolution). Therefore, we have the likelihood for player i as below

$$
\begin{aligned}
L_{i}\left(\Theta_{i} \mid D_{i}, S_{i}\right) & =\prod_{t=1}^{T_{i}} P\left(D_{i t} \mid S_{i t}, \Theta_{i}\right) \times P\left(S_{i, t} \mid S_{i, t-1}, \Theta_{i}\right) \\
& =\prod_{t=1}^{T_{i}} P\left(D_{i t} \mid S_{i t}, \Theta_{i}\right) \times \varphi\left(\frac{\ln A_{i t}-\left(\alpha_{0 i}+\alpha_{1 i} \ln A_{i, t-1}\right)}{\sigma_{i}}\right)
\end{aligned}
$$

where $P\left(D_{i t} \mid S_{i t}, \Theta_{i}\right)$ is from equation (18) or equation (22) under the rational expectation or boundedly rational approach correspondingly.

We model player unobserved heterogeneity by having $\Theta_{i}$ follow a latent class distribution. This is widely known as the latent class approach and adopted in the marketing literature (e.g. Kamakura and Russell 1989). Specifically, we assume $R$ number of latent classes and let $\pi_{r}$ be class weight and $\Theta^{r}$ be the realized values of parameters for class $r$.

We estimated the model parameters using a hierarchical Bayesian approach. To increase the efficiency of the MCMC algorithm, we introduce a latent classification variable $g_{i}$ for each player i, where $g_{i}=r$ if player i belongs to class $r$. The estimation procedure can be divided into three main steps that constitute a "hybrid" algorithm. In the first step, for each player i, conditional on $\left\{\pi_{1}, \ldots, \pi_{R}\right\}$ and $\left\{\Theta^{1}, \ldots, \Theta^{R}\right\}$, we draw latent classification variable $g_{i}$ based on the following full conditional posterior probability.

$P\left(g_{i}=r\right)=\frac{L_{i}\left(\Theta_{i}=\Theta^{r} \mid D_{i}, S_{i}\right) \times \pi_{r}}{\sum_{k=1}^{R} L_{i}\left(\Theta_{i}=\Theta^{k} \mid D_{i}, S_{i}\right) \times \pi_{k}}$

In the second step, we draw $\left\{\pi_{1}, \ldots, \pi_{R}\right\}$ from Dirichlet distribution, $\operatorname{Dir}\left(R, d_{1}+n_{1}, \ldots, d_{R}+n_{R}\right)$, using Gibbs sampling based on $g_{i}$, where $d_{r}$ (set to be 1) is the parameter of prior Dirichlet distribution and $n_{r}$ is the number of players that belong to class $r\left(g_{i}=r\right)$. In the third step, for 
each class $r$, conditional on $g_{i}$, we draw $\Theta^{r}$ using random walk Metropolis-Hastings algorithm based on the following likelihood.

$L^{r}=\prod_{\left\{i: g_{i}=r\right\}} L_{i}\left(\Theta_{i}=\Theta^{r} \mid D_{i}, S_{i}\right)$

We let the MCMC chain run for 40,000 iterations, and used the first 30,000 interactions as burn-in period and the last 10,000 iterations to estimate the posterior means and standard deviations. We adopted two methods to ensure the convergence of the MCMC chain. First, we did the time-series inspection on trace plots, which has been often used in the marketing literature. Second, we conducted the Gelman-Rubin test. Both tests confirmed convergence.

We finally address how we treat the initial condition in constructing the player learning on operation efficiency evolution patterns. For empirical identification, we have followed the learning model literature (Mehta, Rajiv and Srinivasan 2004, and Zhao, Zhao, Helsen 2011) by using observations of $A_{i 1}$ through $A_{i 3}$ to estimate $\widetilde{\alpha}$ for each player and used these estimates as the prior for $\alpha$ for the same individual. We used data from level 4 to level 24 (a total of 21 levels) to estimate the model parameters. With the initial belief on $\widetilde{\alpha}$, each player can form a new belief after completing a level with a new observation of her efficiency, in a deterministic way following the updating equations as explained in the model section.

\section{Findings}

We first discuss our empirical findings and then conduct counterfactual analysis to illustrate the value of our proposed model.

Model Comparison. We first estimated our proposed model under three heterogeneity specifications: one class, two classes, and three classes. We report the model fit statistic (Deviance 
Information Criterion or DIC) and parameter estimates under each of the three specifications in Table 2a. Comparison of DIC suggests that the two-class specification fits the data the best.

$$
==\text { Insert Table } 2 \text { a Here }==
$$

We then test three alternative model specifications under the one-class assumption and under the two-class assumption respectively, with results being reported in Table $2 \mathrm{~b}$ and Table $2 \mathrm{c}$. Our proposed model assumes players to be forward-looking but are boundedly rational in predicting their playing efficiency and utility (as discussed in Section 3.3.2). The first alternative specification turns off the forward-looking aspect, by assuming that players are myopic in their decisions to continue or quit after each level, and they are boundedly rational. The second alternative specification allows forward-looking but assumes that players are fully rational in predicting their playing efficiency and utility (as discussed in Section 3.3.1). The third alternative model assumes that players are forward looking, but instead of behaving according to the proposed bounded rationality, they allow their perceptions to be systemically different from the true means (i.e. $m_{i t}=X_{i t} \mu_{i t}+l$ ). DIC values suggest that our proposed model outperforms the other three models under both the one-class and two-class assumptions. This implies that the data are best explained by a model in which players are forward-looking, but exhibit certain behavioral biases in forming beliefs about how their operation efficiency evolves over game levels. More specifically, we find that while myopic assumption reduces the model fit for about $1 \%$ compared to the proposed model, the full rational approach reduces the model fit for almost $10 \%$, suggesting that the bounded rationality is more important than forward looking in explaining the observed user play-or-quit decisions over the game levels.

$$
==\text { Insert Tables } 2 \mathrm{~b} \text { and } 2 \mathrm{c} \text { Here }==
$$


This two-class specification performs very well in fitting the survival rate at each game level, as shown in Figure 2a. Figure 2 b displays the simulated survival rate at each game level for these two groups of players respectively. As shown, the predicted survival/attrition rate for players in the second class is much higher/lower than that for those in the first class.

$$
==\text { Insert Figures } 2 \mathrm{a} \text { and } 2 \mathrm{~b} \text { Here }^{5}==
$$

Estimation Results. We discuss the parameter estimates from the proposed model with the two-class specification reported in Table $2 \mathrm{a}$, since it produces the best model fit. We find that $78 \%$ of players belong to Class 1 and the remaining $22 \%$ belong to Class 2 . There are important differences in the gameplay preferences of these two groups of players. For convenience, we call players in Class 1 as type 1 and players in Class 2 as type 2.

First, as shown from the estimates of $\gamma_{0}$ and $\gamma_{1}$, type 2 enjoy the game more than type 1 at the beginning, but as they level up, they become satiated with the game more quickly. Second, the estimates of $\varpi_{0}$ and $\varpi_{1}$ tell us that while the marginal cost of playing goes down in a similar rate as players level up for the two types, type 1 have a larger marginal cost than type 2 when they start the game. Third, the estimates of $t_{0}$ and $t_{1}$ reveal to us that the sense of accomplishment (SOA) goes down as type 2 advance to higher levels, but remains stable for type 1 . Fourth, finishing the entire game brings a much higher extra utility for type $2(C=2.169)$ than type $1(C=0.472)$. Fifth, both types are found to be overestimating their operation efficiency since their estimated deviation parameter $l$ is significantly positive with a larger magnitude for type $1(l=2.503)$ than type $2(l=$ 1.789).

\footnotetext{
${ }^{5}$ In Figure 2 and onwards, levels 1-21 are correspondent to Levels 4-24. Since data from Levels 1-3 are used for estimating the initial conditions (priors) for the learning process, we estimated the proposed level-progression model using 21 levels of data from Level 4 to Level 24.
} 
To gain deeper insights on player preferences, we plot in Figures 3a-3d the average utility from operations, cost of operations, SOA, and future values over game levels for the two types of players. The blue solid line represents type 1 players and the orange broken line represents type 2 players.

We find that for type 1 players, their benefit from operation increases gradually but the corresponding cost from operation decreases quickly, suggesting that the net utility from operation plays an important role in their play-or-quit decisions. Meanwhile the SOA and future value are relatively low for type 1 in comparison to type 2, and they decrease over levels. Based on these patterns, we call type 1 players "Experiencers".

As for type 2 players, they get a large benefit from operations at the beginning, however, the benefit vanishes quickly and reaches zero after level 5 . The cost of operation also decreases for type 2. Overall, operations play an important role for type 2 only for the first 3 levels, and after that, the net utility drops to zero (if not negative). The average SOA decreases over levels but is higher for type 2 than for type 1 . Similarly, their future values are also higher. Note that for type 2 players, the future value decreases at the beginning, likely due to the decreasing net utility from operations). However, the future value gradually increases in the second half of the game, likely due to a large extra utility $\mathrm{C}$ from finishing the whole game, as $\mathrm{C}$ becomes less discounted as the player progresses closer toward the end of the game. Given these findings, we believe that the major driver of the continuation decision for type 2 is the sense of achievement, and label type 2 as "Achievers".

In summary, type 1 are players who enjoy the process of playing the game, whereas the type 2 care more about the achievement of finishing the whole game. This is also consistent with our finding of type 2 having a lower predicted attrition. From a managerial standpoint, type 2 are 
the key contributors for the profits of the game company: since they are focused on completing the game, they finish more levels and this longer engagement can bring more revenue to the company.

$$
==\text { Insert Figures } 3 \mathrm{a}, 3 \mathrm{~b}, 3 \mathrm{c} \text { and } 3 \mathrm{~d} \text { Here }==
$$

To gain deeper insights on the dynamics of player learning, we have plotted in Figures 4a$4 d$ the average of perceived mean and variance for the two coefficients of the AR1 process over game levels. Recall that the AR1 process captures the evolution of a player's operation efficiency. Note that $\ln A_{i t}=\alpha_{0 i}+\alpha_{1 i} \ln A_{i, t-1}+\varepsilon_{i t}$ where $\alpha_{0 i}$ can be viewed as the baseline efficiency and $\alpha_{1 i}$ as the carryover. The blue solid line represents type 1 players and the orange broken line represents type 2 players.

There are two main findings. First, the perceived means of both coefficients, converge to their steady states. Second, both perceived variances converge toward zero, but at different speeds. The perceived variance for the intercept decreases much more gradually than that for the slope, suggesting that players are learning the carryover of their operation efficiency faster than learning their baseline operation efficiency. These findings apply to both types of players.

It is important to note that the role of $l_{i}$ will not vanish quickly in the boundedly rational specification for three reasons. First, the perceived variance of the intercept converges toward zero slowly. Second, there is a substantial amount of variation on the $\Sigma_{\text {it }}$ across players. Third, even if the $\Sigma_{\text {it }}$ may be getting close or equal to zero, the perceived variance $\left(\Delta_{i t}^{2}\right)$ will not be zero because $\Delta_{i t}^{2}=X_{i t} \Sigma_{i t} X_{i t}^{\prime}+\sigma_{i}^{2}$, and $\sigma_{i}^{2}$ is always positive.

$$
==\text { Insert Figures } 4 \mathrm{a}, 4 \mathrm{~b}, 4 \mathrm{c} \text { and } 4 \mathrm{~d} \text { Here }==
$$

Robustness Tests. To further validate the model, we have considered several additional specifications. Table $3 \mathrm{a}$ and Table $3 \mathrm{~b}$ report the model fit and parameter estimates from the proposed model and five alternative specifications (Models C1-C5). Model C1 tests an assumption 
that players make unbiased prediction for their operation efficiency, that is, $\ln A_{i t}{ }^{*}=X_{i t} \mu_{i t}$. Model C2 tests an assumption that players make biased prediction, that is, $\ln A_{i t}{ }^{*}=X_{i t} \mu_{i t}+$ constant, and we estimate the constant. Model C3 estimates the proposed model without the additional utility gain from finishing the entire game. Model C4 assumes away higher order interaction terms by setting $\gamma_{l}=\omega_{1}=0$. Model C5 estimates the proposed model under a quadratic utility specification. The fits statistics suggest that our proposed model specification outperforms all the other four alternative specifications, and the main estimation results are robust to these alternative model specifications. We have also tested the quadratic specification on $\beta, \kappa$ and $S O A$ with respect to level $t$, in order to capture the potential satiation of players (Ishihara and Ching 2012). However, the quadratic terms are found to be insignificant.

$$
==\text { Insert Tables } 3 \mathrm{a} \text { and } 3 \mathrm{~b} \text { Here }==
$$

Counterfactual Simulation 1. As discussed earlier, one advantage of the proposed model is its ability to capture consumer risk preferences in a flexible way including risk seeking, risk neutral and risk aversion. As we show in equation 12, the player's sensitivity to uncertainty is a function of the model parameters as well as the state variables. This means, for different players at different levels, their attitudes toward risk are different. To demonstrate the effect of uncertainty on players, we conduct a simulation based on the estimates from our proposed model with two classes. Holding everything else being constant, we change the variance of the error term $\sigma_{i}^{2}$ in the AR1 evolution of the player operation efficiency. For example, the game designer can give some unexpected bonus points during the process to increase the uncertainty. For risk-seeking players, this additional uncertainty may stimulate player interest and engagement. For all players, we simulate the average number of completed levels. Note that, the larger the variance, the more uncertain that a plyer's operation efficiency in level $t$ will be determined by the realized efficiency 
in level t-1, and the larger the player's perceived uncertainty $\left(\Delta_{i t}=\sqrt{\left(X_{i t} \Sigma_{i t} X_{i t}^{\prime}+\sigma_{i}^{2}\right)}\right)$ on the current operation efficiency.

We describe the steps we took for simulating the number of completed levels.

Step 1: Given an uncertainty level, we calculated the value function based on equation $16 \mathrm{~b}$.

Step 2: Since we generated initial state for each player based on the observed efficiency in the first three levels, we used them as the empirical distribution of the initial state. Specifically, for each simulated player $i$, we drew initial states based on this empirical distribution, and then generated simulated player i's initial belief on his/her efficiency according to equation 8 .

Step 3: For simulated player $\mathrm{i}$ at level $\mathrm{t}$, we drew $\eta_{i t}$, and calculated the choice-specific value function according to equation 21, and then generated i's decision at level t.

Step 4: If the generated decision was to quit the game, then simulation ended for i. If the generated decision was to continue the game, we drew $\varepsilon_{i t}$ and calculated efficiency based on equation 4 . Since $A_{i t}=G_{t} / O_{i t}$, the computed efficiency will give us the simulated number of operations $\mathrm{O}_{i t}$. Then we let player $\mathrm{i}$ update her belief on $\left(\alpha_{0 i}, \alpha_{1 i}\right)$ using equations $7 \mathrm{a}-7 \mathrm{c}$ and constructed the player i's belief on efficiency according to equation 8 for the next level.

Results are reported in Table 4. We have two findings. First, on average, we find that the players are risk-seeking, that is, unexpected surprises tend to increase the number of levels played. Second, when the perceived uncertainty gets too large, the players become risk averse. Taken together, an important managerial insight from our analysis is that game developers need to effectively manage user perceived uncertainty on their operation efficiency. The playing utility will increase when additional uncertainty is induced in a reasonable range.

$$
==\text { Insert Table } 4 \text { Here }==
$$


Counterfactual Simulation 2. We next conduct a counterfactual analysis to further demonstrate the use of our model for assisting level design. Level design is an important task for game developers, and the ultimate goal is to maximize some objective function by configuring the optimal level specifications such as cumulative required passing points $\left(H_{t}\right)$ for each game level, where $H_{t}=\sum_{j=1, \ldots, t} G_{j}$. With any one of the two $(H$ and $G)$ given, we can determine the other. For this counterfactual analysis, we propose two new designs, which are reasonably close to the current one, and compare the user engagement (measured by the operation quantity) under alternative designs to identify the potential for improving the current design. As users' operation quantity increases, we anticipate that the game company would have more revenue potential. The assumption is that the game designer can alter the cutoff points within a reasonable range from the status quo to improve user engagement, while maintaining a similar pattern of the operation efficiency evolution.

To propose two candidate new point designs, we first try to empirically identity how the observed cumulative level-progression required points $H_{t}$ vary over levels t. Since $H_{t}$ increases exponentially with respect to level $t$, we postulate the following relationship,

$H_{t}=f_{2} \times\left(e^{f_{0}+f_{1} \times(t-1)}-1\right)$

where $f_{0}, f_{1}$ and $f_{2}$ are coefficients of the function. $f_{0}$ determines the required points to pass the first level that is $H_{1} ; f_{l}$ determines the curvature of this function; and $f_{2}$ determines how fast $H_{t}$ increases. Define $F=\left(f_{0}, f_{1}, f_{2}\right)$, and we find that the estimated $F^{\text {current }}$ with actually observed $H$ is $(0.042$, 0.102 and 4624.312 ) with $\mathrm{R}^{2}$ as 0.999 based on equation 25 . This suggests that the constructed functional form accurately captures how the cumulative required passing points vary over game levels. We assume that a manager will continue to use this function to design the specification of the game. 
Next, we increase/decrease the coefficient of $f_{l}$ (or the curvature of the function of $\mathrm{F}$ ) by $20 \%$, rendering two different counterfactual scenarios: (i) the small curvature scenario $\left(\mathrm{F}^{\text {small }}\right)$, that is, the incremental required passing points $\mathrm{G}_{\mathrm{t}}$ increase more slowly over game levels than in the current design; and (ii) the large curvature scenario $\left(\mathrm{F}^{\text {large }}\right)$, that is, the incremental required passing points $\mathrm{G}_{\mathrm{t}}$ increase more quickly over game levels than in the current design. To make those two new scenarios relatively comparable to the current design, we adjust $f_{2}$ to ensure that the total required number of points to pass all the levels of the game to be the same as observed from the data (i.e. $\mathrm{H}_{24}$ is fixed to be the same as what we observe from the data). Figure $5 \mathrm{a}$ and figure $5 \mathrm{~b}$ plot the cumulative required passing points $\left(\mathrm{H}_{\mathrm{t}}\right)$ and the corresponding incremental required passing points $\left(G_{t}\right)$ at each level, under the three alternative scenarios $\left(F^{\text {current }}, F^{\text {small }}, F^{\text {large }}\right)$. The two new designs are comparable to the current one as shown from the figures.

We then simulate the average total number of operations for the entire sample and for each of the two segments of players under the three point schedules $\left(\mathrm{F}^{\text {current }}, \mathrm{F}^{\text {small }}{ }^{\mathrm{F}}{ }^{\text {large }}\right)$, and report them in Table 5. For the two new designs, we find that $\mathrm{F}^{\text {small }}$ leads to an overall $15.8 \%$ increase of operation quantity, and $\mathrm{F}^{\text {large }}$ leads to an overall $13.5 \%$ decrease of operation quantity, as compared to the current point system $\mathrm{F}^{\text {current }}$. This suggests that the game designer can benefit from allocating more passing points at lower levels and fewer passing points at higher levels compared to the current design.

$$
==\text { Insert Table } 5 \text { Here }==
$$

In the second counterfactual simulation, we simulate the play-or-quit decisions and number of operations under two new point designs. We model the development of operation efficiency $A_{i t}$, which depends on both players' ability and game difficulty at each level. In the counterfactual analysis, we do not change the game difficulty, and instead we change the cutoff points so that the 
player needs to adjust the number of operations to pass a level. For example, imagine that there are 10 similar tasks available at level $\mathrm{t}$, and a player needs to finish 2 tasks in order to pass a level in the previous setup. When the required points increase, players may need to finish 3 tasks to pass the level. Since the game is not a skill-heavy one, more operations at the same level may not lead to a substantial increase in player's ability. Furthermore, available tasks at the same level are similar in terms of their difficulty level, and thus it is less likely for players to gain extra ability by completing more tasks at the same level. We also want to point out that the two new point designs are relatively comparable to the current one, as the total required number of points to pass all the levels of the game remain the same as what is observed from the data (i.e. $\mathrm{H}_{24}$ is fixed to be the same as what we observe from the data). Therefore, changing the cutoff points for level progression under these two similar point designs is unlikely to significantly change the evolution of player efficiency.

\section{Conclusion}

In this paper, we study player level-progression decisions in online gaming where behavioral biases and risk seeking are likely to exist. Players make play-or-quit decisions at each game level and, after completing each level, can observe their realized operation efficiency. We model players' learning about the evolution of the game-playing operation efficiency, and consider two specifications of how players form predictions on their playing efficiency and utility: under rational expectations and under bounded rationality. The proposed boundedly rational approach can flexibly capture players' over/unbiased/under estimation tendencies and risk averse/neutral/seeking preferences, two features that are particularly relevant when modeling the consumption of entertainment products. We cast the individual play-or-quit decisions in a forward- 
looking optimal stopping framework, and develop an algorithm for estimating such dynamic models that incorporate players' bounded rationality. We find the boundedly rational approach substantially outperforms the rational expectation approach.

The managerial contributions of this research are two-fold. First, our study helps game developers better understand users' online gaming behavior. An interesting behavioral construct we have identified in our empirical context relates to how consumers predict their operation efficiency and utility. We have identified two types of game players: "Experiencers" vs. "Achievers". While "Experiencers" derive more utility from playing the game and become satiated more slowly with their marginal return from operations as they advance game levels, "Achievers" are more ambitious and obtain a large boost from finishing the entire game, and hence may be more willing to spend money on "shortcuts" (such as coins or tools within the game) which will speed them towards the finish line.

Second, our proposed modeling framework can help game developers to improve its current level-progression point schedule to more effectively engage players. Game marketers can also adopt our framework to configure surprising bonus points, a common marketing practice promotion in online gaming, based on player's different perceived uncertainty, so that players could play more.

One limitation of the current study relates to data constraints. For each completed level, we observe number of operations and required passing points. For any incomplete level which is the stopping point for a player, we do not know how much the person has played in that level. Furthermore, in the online gaming context, the level-progression decision can be also affected by the type of tasks completed in each level. Since we do not have task-specific information, we cannot study them in our context. Such additional data would allow us to estimate a richer model 
of players' gameplay decisions, potentially focusing not only on level progression but also on task choices within each level. Finally, it is likely that some players are using the boundedly rational decision-making whereas other are using the fully rational decision-making. With more information about individual players, a fruitful future research area is to model such structural heterogeneity. 


\section{References}

Albuquerque, Paulo and Yulia Nevskaya (2012), “The Impact of Innovation on Product Usage: A Dynamic Model with Progression in Content Consumption,"

https://papers.ssrn.com/sol3/papers.cfm?abstract_id=1960020.

Arrow, Kenneth (1962), “The Economic Implications of Learning by Doing," Review of Economic Studies, 29(3), 155-173.

Bernoulli, Daniel (1954), "Exposition of a New Theory on the Measurement of Risk," Econometrica, 22(1), 22-36.

Billeter, Darron, Ajay Kalra, and George Loewenstein (2011), "Underpredicting Learning after Initial Experience with a Product," Journal of Consumer Research, 37(5), 723-736

Brown, J. D., Collins, R. L., and Schmidt, G. W. (1988). "Self-esteem and Direct versus Indirect Forms of Self Enhancement," Journal of Personality and Social Psychology, 55, 445-453.

Buehler, Roger, Dale Griffin, and Michael Ross (1994), “Exploring the 'Planning Fallacy': Why People Underestimate Their Task Completion Times," Journal of Personality and Social Psychology, 67(3), 366-81.

Camerer, C, and D. Lovallo, D. (1999), “Overconfidence and Excess Entry: An Experimental Approach," American Economic Review, 89, 306-318.

Chan, Tat, Jia Li and Lamar Pierce (2014), "Learning from Peers: Knowledge Transfer and Sales Force Productivity Growth,” Marketing Science, 33(4), 463-484.

Ching, Andrew T, Tulin Erdem and Michael Keane (2013), "Learning models: An Assessment of Progress, Challenges and New Developments," Marketing Science, 32(6), 913-938.

Ching, Andrew and Masakazu Ishihara (2012), "Measuring the Informative and Persuasive Roles of Detailing on Prescribing Decisions," Management Science, 58(7), 1374-1387

Chintagunta, P. K., R. Jiang and G. Z. Jin (2009), "Information, Learning, and Drug Diffusion: The Case of Cox-2 Inhibitors," Quantitative Marketing and Economics, 7, 399-343.

Chylinski, Mathew B., John H. Roberts and Bruce G. Hardie (2012), "Consumer Learning of New Binary Attribute Importance Accounting for Priors, Bias, and Order Effects," Marketing Science, 31(4), 549-566. 
Erdem, Tulin and Michael P. Keane (1996), "Decision-Making under Uncertainty: Capturing Choice Dynamics in Turbulent Consumer Goods Markets," Marketing Science, 15(1), 1-20.

Folkes, Valerie (1988), "Recent Attribution Research in Consumer Behavior: A Review and New Directions," Journal of Consumer Research, 14(4), 548-565.

Hartmann, Wes (2006), "Intertemporal Effects of Consumption and Their Implications for Demand Elasticity Estimates," Quantitative Marketing and Economics, 4(4).

Hirshleifer, D. and Luo, G. Y. (2001), "On the Survival of Overconfident Traders in a Competitive Securities Market. Journal of Financial Markets, 4(1), 73-84.

Huang, Yan, Stefanus Jasin, and Puneet Manchanda (2019), ““'Level Up”: Leveraging Skill and Engagement to Maximize Player Retention in Online Video Games," Information Systems Research, 30(3), 927-947.

Ishihara, Masakazu and Andrew Ching (2012), "Dynamic Demand for New and Used Durable Goods without Physical Depreciation: The Case of Japanese Video Games," Rotman School of Management Working Paper No. 2189871. Available at: http://dx.doi.org/10.2139/ssrn.2189871

Kamakura, Wagner A. and Gary J. Russell (1989), "A Probabilistic Choice Model for Market Segmentation and Elasticity Structure,” Journal of Marketing Research, 26(4), 379-390.

Kim, Young-Hoon, Heewon Kwon, Joo Lee, and Chi-Yue Chiu (2016), "Why Do People Overestimate or Underestimate Their Abilities? A Cross-Culturally Valid Model of Cognitive and Motivational Processes in Self-Assessment Biases," Journal of Cross-Cultural Psychology, 47(9) 1201-1216.

Kruglanski, A. W., \& Webster, D. M. (1996), "Motivated Closing of the Mind: "Seizing" and "Freezing"," Psychological Review, 103(2), 263-283.

Langer, Ellen J. (1975), "The Illusion of Control," The Journal of Personality and Social Psychology, 32(2), 311-328.

Lee, Clarence, Vineet Kumar and Sunil Gupta (2014), "Designing Freemium: a Model of Consumer Usage, Upgrade, and Referral Dynamics," Working Paper, Cornell University.

Libby, Robert and Kristina Rennekamp (2012), "Self-Serving Attribution Bias, Overconfidence, and the Issuance of Management Forecasts," Journal of Accounting Research, 50(1), 197-230.

Nevskaya, Yulia and Paulo Albuquerque (2019), "How Should Firms Manage Excessive Product Use? A Continuous-Time Demand Model to Test Reward Schedules, Notifications, and Time Limits," Journal of Marketing Research, 56(3), 379-400 
Weinstein, Neil D. (1989), “Optimistic Biases about Personal Risks,” Science, 246, 1232-33.

Mehta, N., S. Rajiv and K. Srinivasan (2003), "Price Uncertainty and Consumer Search: A Structural Model of Consideration Set Formation," Marketing Science, 22(1), 40-57.

Oskamp, Stuart (1965), "Overconfidence in Case-Study Judgments," Journal of Consulting Psychology, 29(3), 261-65.

Rust, John (1988), "Maximum Likelihood Estimation of Discrete Control Processes." SIAM Journal on Control and Optimization, 26(5), 1006-1024.

Taylor, S. E., and J. D. Brown (1988), "Illusion and Well-being: A Social Psychological Perspective on Mental Health. Psychological Bulletin, 103, 193-210.

Zauberman, Gal and John G. Lynch Jr. (2005), "Resource Slack and Propensity to Discount Delayed Investments of Time Versus Money," Journal of Experimental Psychology: General, 134(1), 23-37.

Zhao, Yi, Zhao Ying and Helsen Kristiaan, (2011) "Consumer Learning in a Turbulent Market Environment: Modeling Consumer Choice Dynamics after a Product Harm Crisis," Journal of Marketing Research, 48(2), 255-267.

Zhao, Yi, Sha Yang, Vishal Narayan and Ying Zhao (2013), "Modeling Consumer Learning from Online Product Reviews," Marketing Science, 32(1), 153-169.

Zuckerman, Marvin, 1994, Behavioral Expression and Biosocial Bases of Sensation Seeking, Cambridge University Press, New York, NY. 
Table 1a. Mean summary statistics

\begin{tabular}{|c|c|c|c|c|c|c|c|}
\hline Level & $\begin{array}{r}\text { Required } \\
\text { Point }\end{array}$ & $\begin{array}{r}\text { Operation } \\
\text { Quantity }\end{array}$ & $\begin{array}{l}\text { Operation } \\
\text { Efficiency }\end{array}$ & $\begin{array}{r}\text { Attrition } \\
\text { Rate }\end{array}$ & $\begin{array}{r}\text { Operation } \\
\text { Quantity }\end{array}$ & $\begin{array}{l}\text { Operation } \\
\text { Efficiency }\end{array}$ & $\begin{array}{r}\text { Attrition } \\
\text { Rate }\end{array}$ \\
\hline 1 & 8 & 3.304 & 2.535 & & \multirow[t]{4}{*}{13.468} & \multirow[t]{4}{*}{14.302} & \multirow[t]{4}{*}{0.204} \\
\hline 2 & 42 & 15.341 & 3.568 & & & & \\
\hline 3 & 100 & 17.186 & 15.611 & & & & \\
\hline 4 & 200 & 18.040 & 35.495 & 0.204 & & & \\
\hline 5 & 300 & 21.142 & 42.136 & 0.178 & \multirow[t]{5}{*}{30.627} & \multirow[t]{5}{*}{47.505} & \multirow[t]{5}{*}{0.162} \\
\hline 6 & 430 & 26.846 & 43.875 & 0.145 & & & \\
\hline 7 & 600 & 32.209 & 58.570 & 0.163 & & & \\
\hline 8 & 720 & 36.374 & 41.001 & 0.152 & & & \\
\hline 9 & 900 & 36.562 & 51.945 & 0.174 & & & \\
\hline 10 & 1000 & 35.946 & 49.972 & 0.139 & \multirow[t]{5}{*}{36.536} & \multirow[t]{5}{*}{63.562} & \multirow[t]{5}{*}{0.154} \\
\hline 11 & 1000 & 37.338 & 64.925 & 0.168 & & & \\
\hline 12 & 1100 & 32.415 & 60.675 & 0.151 & & & \\
\hline 13 & 1300 & 36.173 & 73.659 & 0.169 & & & \\
\hline 14 & 1400 & 40.810 & 68.580 & 0.143 & & & \\
\hline 15 & 1500 & 44.154 & 60.090 & 0.071 & \multirow[t]{5}{*}{48.158} & \multirow[t]{5}{*}{64.689} & \multirow[t]{5}{*}{0.149} \\
\hline 16 & 1500 & 37.286 & 71.398 & 0.103 & & & \\
\hline 17 & 1800 & 46.102 & 65.160 & 0.157 & & & \\
\hline 18 & 2000 & 58.087 & 54.674 & 0.220 & & & \\
\hline 19 & 2100 & 55.162 & 72.123 & 0.196 & & & \\
\hline 20 & 2200 & 49.483 & 93.799 & 0.216 & \multirow[t]{5}{*}{54.136} & \multirow[t]{5}{*}{84.803} & \multirow[t]{5}{*}{0.103} \\
\hline 21 & 2600 & 58.862 & 87.362 & 0.000 & & & \\
\hline 22 & 2700 & 57.167 & 69.729 & 0.172 & & & \\
\hline 23 & 2800 & 50.455 & 73.112 & 0.083 & & & \\
\hline 24 & 2900 & 54.714 & 100.013 & 0.045 & & & \\
\hline
\end{tabular}


Table 1b. Summary statistics of operation quantity

\begin{tabular}{|c|r|r|r|r|}
\hline Level & Mean & Std. Dev. & Min & Max \\
\hline 1 & 3.304 & 0.803 & 3.000 & 7.000 \\
\hline 2 & 15.341 & 6.538 & 5.000 & 38.000 \\
\hline 3 & 17.186 & 14.221 & 2.000 & 67.000 \\
\hline 4 & 18.040 & 18.348 & 2.000 & 125.000 \\
\hline 5 & 21.142 & 21.747 & 2.000 & 155.000 \\
\hline 6 & 26.846 & 25.568 & 3.000 & 171.000 \\
\hline 7 & 32.209 & 29.222 & 2.000 & 221.000 \\
\hline 8 & 36.374 & 30.043 & 6.000 & 218.000 \\
\hline 9 & 36.562 & 27.291 & 6.000 & 171.000 \\
\hline 10 & 35.946 & 30.302 & 8.000 & 215.000 \\
\hline 11 & 37.338 & 38.898 & 3.000 & 326.000 \\
\hline 12 & 32.415 & 22.430 & 9.000 & 136.000 \\
\hline 13 & 36.173 & 24.147 & 3.000 & 100.000 \\
\hline 14 & 40.810 & 27.475 & 5.000 & 124.000 \\
\hline 15 & 44.154 & 33.403 & 7.000 & 221.000 \\
\hline 16 & 37.286 & 21.107 & 10.000 & 116.000 \\
\hline 17 & 46.102 & 30.007 & 10.000 & 151.000 \\
\hline 18 & 58.087 & 48.602 & 14.000 & 283.000 \\
\hline 19 & 55.162 & 44.810 & 9.000 & 250.000 \\
\hline 20 & 49.483 & 30.731 & 4.000 & 138.000 \\
\hline 21 & 58.862 & 35.728 & 7.000 & 135.000 \\
\hline 22 & 57.167 & 37.064 & 18.000 & 174.000 \\
\hline 23 & 50.455 & 30.671 & 22.000 & 133.000 \\
\hline 24 & 54.714 & 33.956 & 12.000 & 114.000 \\
\hline & & & & \\
\hline
\end{tabular}


Table 1c. Summary statistics of operation efficiency

\begin{tabular}{|c|r|r|r|r|}
\hline Level & Mean & Std. Dev. & Min & Max \\
\hline 1 & 2.535 & 0.560 & 1.143 & 2.667 \\
\hline 2 & 3.568 & 2.806 & 1.105 & 8.400 \\
\hline 3 & 15.611 & 21.689 & 1.493 & 50.000 \\
\hline 4 & 35.495 & 51.086 & 1.600 & 200.000 \\
\hline 5 & 42.136 & 63.314 & 1.935 & 150.000 \\
\hline 6 & 43.875 & 74.252 & 2.515 & 143.333 \\
\hline 7 & 58.570 & 115.557 & 2.715 & 300.000 \\
\hline 8 & 41.001 & 54.561 & 3.303 & 120.000 \\
\hline 9 & 51.945 & 75.118 & 5.263 & 150.000 \\
\hline 10 & 49.972 & 55.083 & 4.651 & 125.000 \\
\hline 11 & 64.925 & 98.262 & 3.067 & 333.333 \\
\hline 12 & 60.675 & 85.336 & 8.088 & 122.222 \\
\hline 13 & 73.659 & 104.583 & 13.000 & 433.333 \\
\hline 14 & 68.580 & 106.348 & 11.290 & 280.000 \\
\hline 15 & 60.090 & 67.035 & 6.787 & 214.286 \\
\hline 16 & 71.398 & 108.849 & 12.931 & 150.000 \\
\hline 17 & 65.160 & 72.450 & 11.921 & 180.000 \\
\hline 18 & 54.674 & 37.823 & 7.067 & 142.857 \\
\hline 19 & 72.123 & 77.676 & 8.400 & 233.333 \\
\hline 20 & 93.799 & 132.734 & 15.942 & 550.000 \\
\hline 21 & 87.362 & 110.091 & 19.259 & 371.429 \\
\hline 22 & 69.729 & 45.487 & 15.517 & 150.000 \\
\hline 23 & 73.112 & 35.778 & 21.053 & 127.273 \\
\hline 24 & 100.013 & 122.734 & 25.439 & 241.667 \\
\hline & & & & \\
\hline & & & & \\
\hline 13 & & & & \\
\hline & & & &
\end{tabular}


Table 2a. Proposed model with different number of latent class

\begin{tabular}{|c|c|c|c|c|c|c|c|c|c|c|c|c|}
\hline & \multicolumn{2}{|c|}{ Model - One Class } & \multicolumn{4}{|c|}{ Model - Two Classes (Best Fit) } & \multicolumn{6}{|c|}{ Model - Three Classes } \\
\hline $\begin{array}{c}\text { Log Marginal } \\
\text { Likelihood }\end{array}$ & -2751.976 & & -2633.42 & & & & -2630.1 & & & & & \\
\hline No. of Parameters & 11 & & 23 & & & & 35 & & & & & \\
\hline DIC: & 5503.666 & & 5263.828 & & & & 5267.846 & & & & & \\
\hline & Class 1 & & Class 1 & & Class 2 & & Class 1 & & Class 2 & & Class 3 & \\
\hline & Estimate & Std. Err. & Estimate & Std. Err. & Estimate & Std. Err. & Estimate & Std. Err. & Estimate & Std. Err. & Estimate & Std. Err. \\
\hline Percent_of_Class & NA & NA & 0.783 & 0.025 & NA & NA & 0.296 & 0.042 & 0.017 & 0.005 & NA & NA \\
\hline$\gamma_{0}$ & -1.539 & 0.150 & -1.850 & 0.157 & 0.961 & 0.047 & -2.301 & 0.125 & 0.578 & 1.485 & -1.733 & 0.114 \\
\hline$\gamma_{1}$ & -0.017 & 0.032 & -0.020 & 0.071 & -0.713 & 0.057 & 0.044 & 0.020 & 0.560 & 0.292 & -0.704 & 0.111 \\
\hline$\omega_{0}$ & -2.145 & 0.071 & -1.884 & 0.072 & -2.946 & 0.043 & -2.715 & 0.067 & -2.689 & 1.251 & -2.495 & 0.079 \\
\hline$\omega_{1}$ & -0.157 & 0.034 & -0.214 & 0.051 & -0.248 & 0.036 & -0.224 & 0.042 & -0.028 & 0.168 & -0.251 & 0.037 \\
\hline$t_{0}$ & 0.028 & 0.029 & -0.026 & 0.041 & 0.361 & 0.041 & 0.376 & 0.077 & -5.505 & 2.796 & -0.037 & 0.025 \\
\hline $\mathrm{t}_{1}$ & -0.011 & 0.006 & -0.007 & 0.010 & -0.025 & 0.003 & -0.050 & 0.010 & 0.007 & 0.172 & 0.002 & 0.003 \\
\hline$C$ & 1.019 & 0.116 & 0.472 & 0.069 & 2.619 & 0.032 & 0.957 & 0.102 & 12.388 & 3.228 & 1.461 & 0.142 \\
\hline$l$ & 2.450 & 0.089 & 2.503 & 0.087 & 1.789 & 0.070 & 2.101 & 0.180 & 0.255 & 1.266 & 2.163 & 0.110 \\
\hline$\sigma^{2}$ & 0.831 & 0.022 & 1.119 & 0.046 & 0.418 & 0.021 & 0.441 & 0.027 & 0.445 & 0.094 & 1.185 & 0.054 \\
\hline$\alpha_{0}$ & 2.166 & 0.056 & 2.544 & 0.094 & 1.460 & 0.090 & 1.560 & 0.099 & 1.844 & 0.276 & 2.667 & 0.136 \\
\hline$\alpha_{1}$ & 0.385 & 0.016 & 0.277 & 0.026 & 0.592 & 0.026 & 0.546 & 0.029 & 0.553 & 0.064 & 0.249 & 0.033 \\
\hline
\end{tabular}


Table 2b. Model comparison by specification (one class - without heterogeneity)

\begin{tabular}{|c|c|c|c|c|c|c|c|c|}
\hline \multirow{2}{*}{$\begin{array}{c}\text { Log Marginal } \\
\text { Likelihood }\end{array}$} & \multicolumn{2}{|c|}{$\begin{array}{c}\text { Proposed Model - } \\
\text { Forward Looking and } \\
\text { Boundedly Rational }\end{array}$} & \multicolumn{2}{|c|}{$\begin{array}{c}\text { Model A1 - } \\
\text { Myopic and Boundedly } \\
\text { Rational }\end{array}$} & \multicolumn{2}{|c|}{$\begin{array}{c}\text { Model A2 - } \\
\text { Forward Looking and } \\
\text { Fully Rational }\end{array}$} & \multicolumn{2}{|c|}{$\begin{array}{c}\text { Model A3 - } \\
\text { Forward Looking, Biased } \\
\text { Perceived Mean, and } \\
\text { Fully Rational }\end{array}$} \\
\hline & -2751.976 & & -2768.933 & & -3086.040 & & -3057.4 & \\
\hline No. of Parameters & 11 & & 10 & & 10 & & 11 & \\
\hline DIC: & 5503.666 & & 5537.924 & & 6047.047 & & 5842.397 & \\
\hline & Estimate & Std. Err. & Estimate & Std. Err. & Estimate & Std. Err. & Estimate & Std. Err. \\
\hline Percent_of_Class & NA & NA & NA & NA & NA & NA & NA & NA \\
\hline$\gamma_{0}$ & -1.539 & 0.150 & -1.350 & 0.062 & -5.735 & 0.087 & -5.852 & 0.107 \\
\hline$\gamma_{1}$ & -0.017 & 0.032 & -0.221 & 0.030 & 0.068 & 0.051 & 0.085 & 0.085 \\
\hline$\omega_{0}$ & -2.145 & 0.071 & -3.282 & 0.142 & -7.773 & 0.554 & -7.501 & 0.42 \\
\hline$\omega_{1}$ & -0.157 & 0.034 & -0.261 & 0.025 & -0.285 & 0.020 & -0.284 & 0.014 \\
\hline $\mathrm{t}_{0}$ & 0.028 & 0.029 & -0.005 & 0.022 & 0.043 & 0.018 & 0.035 & 0.021 \\
\hline $\mathrm{t}_{1}$ & -0.011 & 0.006 & 0.012 & 0.002 & -0.004 & 0.003 & -0.002 & 0.003 \\
\hline$C$ & 1.019 & 0.116 & NA & NA & 1.230 & 0.087 & 1.168 & 0.052 \\
\hline$l$ & 2.450 & 0.089 & 1.129 & 0.057 & NA & NA & 1.992 & 0.489 \\
\hline$\sigma^{2}$ & 0.831 & 0.022 & 0.831 & 0.023 & 0.816 & 0.025 & 0.807 & 0.022 \\
\hline$\alpha_{0}$ & 2.166 & 0.056 & 2.166 & 0.055 & 2.166 & 0.055 & 2.166 & 0.055 \\
\hline$\alpha_{1}$ & 0.385 & 0.016 & 0.385 & 0.016 & 0.384 & 0.016 & 0.385 & 0.016 \\
\hline
\end{tabular}


Table 2c. Model comparison by specification (two classes - with heterogeneity)

\begin{tabular}{|c|r|r|r|r|r|r|r|r|}
\hline & \multicolumn{3}{|c|}{ Proposed Model - } & \multicolumn{5}{c|}{ Model B1 - } \\
\hline $\begin{array}{c}\text { Log Marginal } \\
\text { Likelihood }\end{array}$ & -2633.417 & & & & -2667.498 & & & \\
\hline No. of Parameters & 23 & & & & 21 & & & \\
\hline DIC: & 5263.828 & & & & 5338.341 & & & \\
\hline & Class 1 & & Class 2 & & Class 1 & & Class 2 & \\
\hline & Estimate & Std. Err. & Estimate & Std. Err. & Estimate & Std. Err. & Estimate & Std. Err. \\
\hline Percent_of_Class & 0.783 & 0.025 & NA & NA & 0.876 & 0.018 & NA & NA \\
\hline$\gamma_{0}$ & -1.850 & 0.157 & 0.961 & 0.047 & -2.197 & 0.076 & 2.021 & 0.055 \\
\hline$\gamma_{1}$ & -0.020 & 0.071 & -0.713 & 0.057 & 0.049 & 0.014 & -0.260 & 0.013 \\
\hline$\omega_{0}$ & -1.884 & 0.072 & -2.946 & 0.043 & -3.288 & 0.100 & -1.818 & 0.046 \\
\hline$\omega_{1}$ & -0.214 & 0.051 & -0.248 & 0.036 & -0.175 & 0.031 & -0.258 & 0.021 \\
\hline $\mathrm{t}_{0}$ & -0.026 & 0.041 & 0.361 & 0.041 & 0.164 & 0.031 & -2.863 & 0.185 \\
\hline $\mathrm{t}_{1}$ & -0.007 & 0.010 & -0.025 & 0.003 & -0.040 & 0.008 & 0.170 & 0.009 \\
\hline$C$ & 0.472 & 0.069 & 2.619 & 0.032 & NA & NA & NA & NA \\
\hline$l$ & 2.503 & 0.087 & 1.789 & 0.070 & 1.151 & 0.037 & -0.037 & 0.057 \\
\hline$\sigma^{2}$ & 1.119 & 0.046 & 0.418 & 0.021 & 1.005 & 0.028 & 0.396 & 0.022 \\
\hline$\alpha_{0}$ & 2.544 & 0.094 & 1.460 & 0.090 & 2.412 & 0.074 & 1.341 & 0.107 \\
\hline$\alpha_{1}$ & 0.277 & 0.026 & 0.592 & 0.026 & 0.306 & 0.021 & 0.637 & 0.030 \\
\hline
\end{tabular}


Table 2c (continued). Model comparison by specification (two classes - with heterogeneity)

\begin{tabular}{|c|c|c|c|c|c|c|c|c|}
\hline \multirow{2}{*}{$\begin{array}{l}\text { Log Marginal } \\
\text { Likelihood }\end{array}$} & \multicolumn{4}{|c|}{$\begin{array}{c}\text { Model B2 - } \\
\text { Forward Looking and Fully Rational }\end{array}$} & \multicolumn{4}{|c|}{$\begin{array}{c}\text { Model B3 - } \\
\text { Forward Looking, Biased Perceived Mean, } \\
\text { and Fully Rational }\end{array}$} \\
\hline & -2951.541 & & & & -2821.726 & & & \\
\hline No. of Parameters & 21 & & & & 23 & & & \\
\hline \multirow[t]{3}{*}{ DIC: } & 5750.371 & & & & 5465.286 & & & \\
\hline & Class 1 & & Class 2 & & Class 1 & & Class 2 & \\
\hline & Estimate & $\begin{array}{l}\text { Std. } \\
\text { Err. }\end{array}$ & Estimate & $\begin{array}{l}\text { Std. } \\
\text { Err. }\end{array}$ & Estimate & $\begin{array}{l}\text { Std. } \\
\text { Err. }\end{array}$ & Estimate & $\begin{array}{l}\text { Std. } \\
\text { Err. }\end{array}$ \\
\hline Percent_of_Class & 0.307 & 0.061 & NA & $\mathrm{NA}$ & 0.301 & 0.044 & NA & NA \\
\hline$\gamma_{0}$ & -5.460 & 0.330 & -6.083 & 0.147 & -5.676 & 0.138 & -5.494 & 0.184 \\
\hline$\gamma_{1}$ & 0.007 & 0.063 & 0.095 & 0.072 & -0.268 & 0.108 & -0.208 & 0.097 \\
\hline$\omega_{0}$ & -8.378 & 0.797 & -8.071 & 0.399 & -6.009 & 0.103 & -8.559 & 0.711 \\
\hline$\omega_{1}$ & -0.277 & 0.022 & -0.258 & 0.036 & -0.211 & 0.052 & -0.268 & 0.031 \\
\hline $\mathrm{t}_{0}$ & 0.247 & 0.088 & -0.019 & 0.037 & 0.184 & 0.043 & 0.006 & 0.024 \\
\hline $\mathrm{t}_{1}$ & -0.018 & 0.006 & -0.004 & 0.006 & -0.010 & 0.004 & -0.002 & 0.003 \\
\hline$C$ & 1.884 & 0.111 & 1.166 & 0.076 & 1.573 & 0.167 & 1.362 & 0.113 \\
\hline$l$ & NA & NA & NA & $\mathrm{NA}$ & 0.644 & 0.109 & 2.549 & 0.175 \\
\hline$\sigma^{2}$ & 0.443 & 0.029 & 1.177 & 0.071 & 0.418 & 0.025 & 1.085 & 0.046 \\
\hline$\alpha_{0}$ & 1.630 & 0.111 & 2.540 & 0.136 & 1.413 & 0.089 & 2.626 & 0.112 \\
\hline$\alpha_{1}$ & 0.540 & 0.032 & 0.282 & 0.035 & 0.602 & 0.026 & 0.261 & 0.030 \\
\hline
\end{tabular}


Table 3a. Model robustness check

\begin{tabular}{|c|c|c|c|c|c|c|c|c|c|c|c|c|}
\hline \multirow[b]{2}{*}{ Log Marginal Likelihood } & \multicolumn{4}{|c|}{ Proposed Model } & \multicolumn{4}{|c|}{ Model C1 - Unbiased Prediction } & \multicolumn{4}{|c|}{ Model C2 - Mean + Constant } \\
\hline & -2633.417 & & & & -2644.781 & & & & -2637.322 & & & \\
\hline No. of Parameters & 23 & & & & 21 & & & & 23 & & & \\
\hline DIC: & 5263.828 & & & & 5279.351 & & & & 5267.590 & & & \\
\hline & Class 1 & & Class 2 & & Class 1 & & Class 2 & & Class 1 & & Class 2 & \\
\hline & Estimate & Std. Err. & Estimate & Std. Err. & Estimate & Std. Err. & Estimate & Std. Err. & Estimate & Std. Err. & Estimate & Std. Err. \\
\hline Percent_of_Class & 0.783 & 0.025 & 0.217 & 0.025 & 0.761 & 0.024 & NA & NA & 0.755 & 0.032 & NA & NA \\
\hline$\gamma_{0}$ & -1.850 & 0.157 & 0.961 & 0.047 & -2.593 & 0.120 & 0.722 & 0.072 & -2.819 & 0.099 & 0.208 & 0.057 \\
\hline$\gamma_{1}$ & -0.020 & 0.071 & -0.713 & 0.057 & 0.049 & 0.018 & -0.659 & 0.077 & 0.042 & 0.034 & -0.581 & 0.106 \\
\hline$\omega_{0}$ & -1.884 & 0.072 & -2.946 & 0.043 & -4.831 & 0.123 & -4.003 & 0.102 & -3.457 & 0.171 & -2.989 & 0.056 \\
\hline$\omega_{1}$ & -0.214 & 0.051 & -0.248 & 0.036 & -0.165 & 0.030 & -0.283 & 0.013 & -0.279 & 0.015 & -0.271 & 0.025 \\
\hline$t_{0}$ & -0.026 & 0.041 & 0.361 & 0.041 & -0.022 & 0.043 & 0.279 & 0.047 & 0.037 & 0.060 & 0.220 & 0.042 \\
\hline $\mathrm{t}_{1}$ & -0.007 & 0.010 & -0.025 & 0.003 & -0.039 & 0.013 & -0.017 & 0.004 & -0.025 & 0.014 & -0.012 & 0.003 \\
\hline$C$ & 0.472 & 0.069 & 2.619 & 0.032 & 0.276 & 0.100 & 2.040 & 0.100 & 1.358 & 0.046 & 1.560 & 0.090 \\
\hline$l$ & 2.503 & 0.087 & 1.789 & 0.070 & NA & NA & NA & NA & 1.212 & 0.082 & 1.237 & 0.065 \\
\hline$\sigma^{2}$ & 1.119 & 0.046 & 0.418 & 0.021 & 1.197 & 0.049 & 0.431 & 0.018 & 1.141 & 0.037 & 0.429 & 0.022 \\
\hline$\alpha_{0}$ & 2.544 & 0.094 & 1.460 & 0.090 & 2.597 & 0.095 & 1.485 & 0.082 & 2.607 & 0.104 & 1.466 & 0.081 \\
\hline$\alpha_{1}$ & 0.277 & 0.026 & 0.592 & 0.026 & 0.265 & 0.026 & 0.582 & 0.023 & 0.263 & 0.028 & 0.588 & 0.023 \\
\hline
\end{tabular}


Table 3b. Model robustness check (continued)

\begin{tabular}{|c|c|c|c|c|c|c|c|c|c|c|c|c|}
\hline \multirow[b]{2}{*}{ Log Marginal Likelihood } & \multicolumn{4}{|c|}{ Model C3 - No C } & \multicolumn{4}{|c|}{ Model C4 - No Interaction } & \multicolumn{4}{|c|}{ Model C5 - Quadratic } \\
\hline & -2644.609 & & & & -2641.960 & & & & -2660.407 & & & \\
\hline No. of Parameters & 21 & & & & 15 & & & & 21 & & & \\
\hline DIC: & 5290.595 & & & & 5284.107 & & & & 5318.758 & & & \\
\hline & Class 1 & & Class 2 & & Class 1 & & Class 2 & & Class 1 & & Class 2 & \\
\hline & Estimate & Std. Err. & Estimate & Std. Err. & Estimate & Std. Err. & Estimate & Std. Err. & Estimate & Std. Err. & Estimate & Std. Err. \\
\hline Percent_of_Class & 0.748 & 0.032 & NA & NA & 0.604 & 0.046 & NA & NA & 0.793 & 0.028 & NA & NA \\
\hline$\gamma_{0}$ & -2.214 & 0.113 & 1.350 & 0.086 & -2.943 & 0.557 & -2.835 & 0.166 & -2.835 & 0.185 & 0.118 & 0.060 \\
\hline$\gamma_{1}$ & 0.044 & 0.018 & -0.808 & 0.089 & -0.288 & 0.359 & 0.072 & 0.409 & -0.966 & 0.026 & -0.948 & 0.049 \\
\hline$\omega_{0}$ & -2.515 & 0.071 & -3.563 & 0.094 & -3.642 & 0.444 & -4.572 & 0.231 & -4.904 & 0.073 & -3.951 & 0.040 \\
\hline$\omega_{1}$ & -0.205 & 0.034 & -0.145 & 0.043 & 0.010 & 0.169 & 0.043 & 0.190 & -0.258 & 0.044 & -0.266 & 0.027 \\
\hline$t_{0}$ & 0.029 & 0.041 & -0.001 & 0.050 & -0.025 & 0.021 & 0.025 & 0.019 & -0.099 & 0.025 & 0.264 & 0.081 \\
\hline $\mathrm{t}_{1}$ & -0.022 & 0.009 & 0.010 & 0.004 & 0.049 & 0.152 & -0.007 & 0.154 & 0.007 & 0.003 & -0.020 & 0.006 \\
\hline$C$ & NA & NA & NA & NA & 1.444 & 0.323 & 1.441 & 0.254 & 0.036 & 0.079 & 2.094 & 0.075 \\
\hline$l$ & 1.740 & 0.068 & 2.313 & 0.054 & 2.184 & 0.257 & 2.432 & 0.141 & 2.575 & 0.072 & 2.569 & 0.059 \\
\hline$\sigma^{2}$ & 1.128 & 0.058 & 0.416 & 0.023 & 1.199 & 0.055 & 0.441 & 0.021 & 1.212 & 0.046 & 0.420 & 0.019 \\
\hline$\alpha_{0}$ & 2.591 & 0.099 & 1.461 & 0.081 & 2.734 & 0.123 & 1.478 & 0.082 & 2.344 & 0.083 & 1.746 & 0.087 \\
\hline$\alpha_{1}$ & 0.268 & 0.027 & 0.590 & 0.024 & 0.235 & 0.032 & 0.581 & 0.023 & 0.322 & 0.024 & 0.518 & 0.025 \\
\hline
\end{tabular}


Table 4 . The first counterfactual analysis by changing variance $\sigma^{2}$

\begin{tabular}{|c|r|r|r|}
\hline \multicolumn{4}{|l|}{ Simulated Number of Levels Played } \\
\hline Change of $\sigma^{2}$ & Overall & Class 1 (Experiencers) & Class 2 (Achievers) \\
\hline-0.400 & 5.375 & 4.255 & 9.118 \\
\hline-0.200 & 5.547 & 4.320 & 9.648 \\
\hline 0.000 & 5.703 & 4.364 & 10.176 \\
\hline 0.200 & 5.778 & 4.396 & 10.395 \\
\hline 0.400 & 5.831 & 4.417 & 10.554 \\
\hline 1.000 & 5.900 & 4.446 & 10.761 \\
\hline 2.000 & 5.925 & 4.455 & 10.833 \\
\hline 3.000 & 5.922 & 4.455 & 10.823 \\
\hline 4.000 & 5.916 & 4.454 & 10.800 \\
\hline
\end{tabular}

Table 5. The second counterfactual analysis for improving the current cutoff point design

\begin{tabular}{|l|c|c|c|}
\hline & $\begin{array}{c}\text { Current Design } \\
\left(\mathrm{F}^{\text {current }}\right)\end{array}$ & $\begin{array}{c}\text { Small Curvature } \\
\text { Design }\left(\mathrm{F}^{\text {small }}\right)\end{array}$ & $\begin{array}{c}\text { Large Curvature } \\
\text { Design }\left(\mathrm{F}^{\text {large }}\right)\end{array}$ \\
\hline$f_{0}$ (initial level coefficient) & 0.042 & 0.042 & 0.042 \\
\hline$f_{1}$ (curvature coefficient) & 0.102 & $0.081(-20 \%)$ & $0.122(+20 \%)$ \\
\hline$f_{2}$ (slope coefficient) & 4624.312 & 7480.619 & 2939.540 \\
\hline Operations - Overall & 207.653 & $240.453(+15.8 \%)$ & $179.537(-13.5 \%)$ \\
\hline Operations - Class 1 (Experiencers) & 141.472 & $170.102(+20.2 \%)$ & $117.139(-17.2 \%)$ \\
\hline Operations - Class 2 (Achievers) & 447.138 & $495.030(+10.7 \%)$ & $405.336(-9.3 \%)$ \\
\hline
\end{tabular}


Figure 1. Average operating efficiency of different groups of players

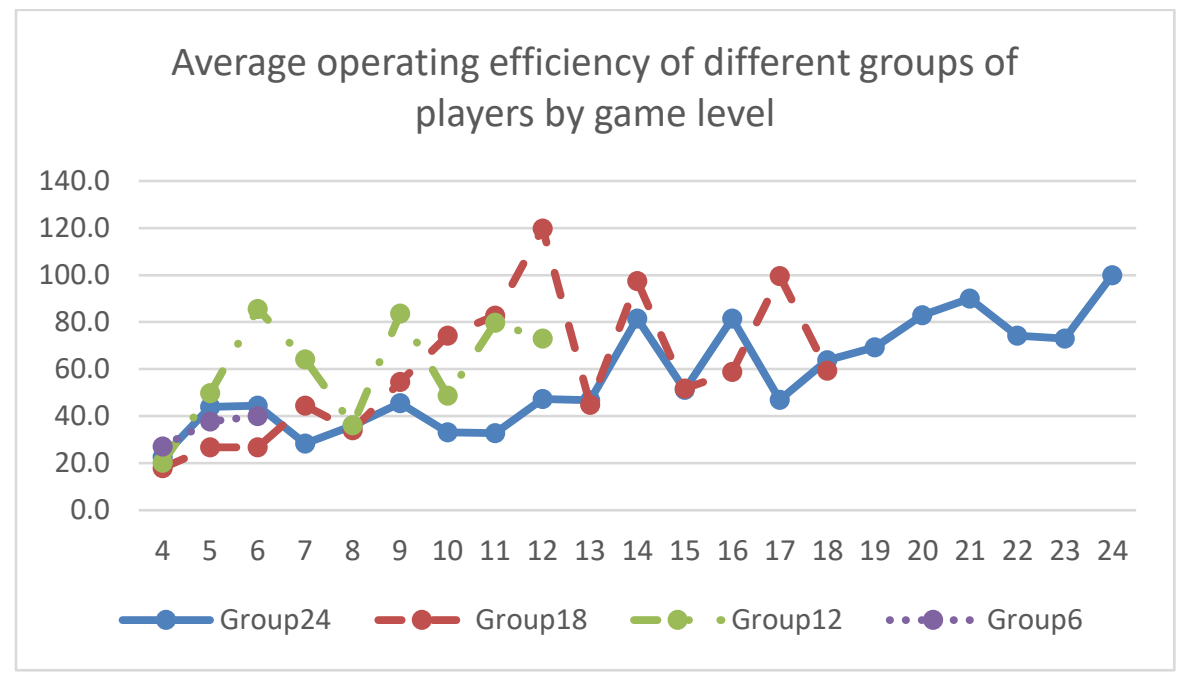


Figure $2 \mathrm{a}$. Observed and fitted overall survival rate by game level

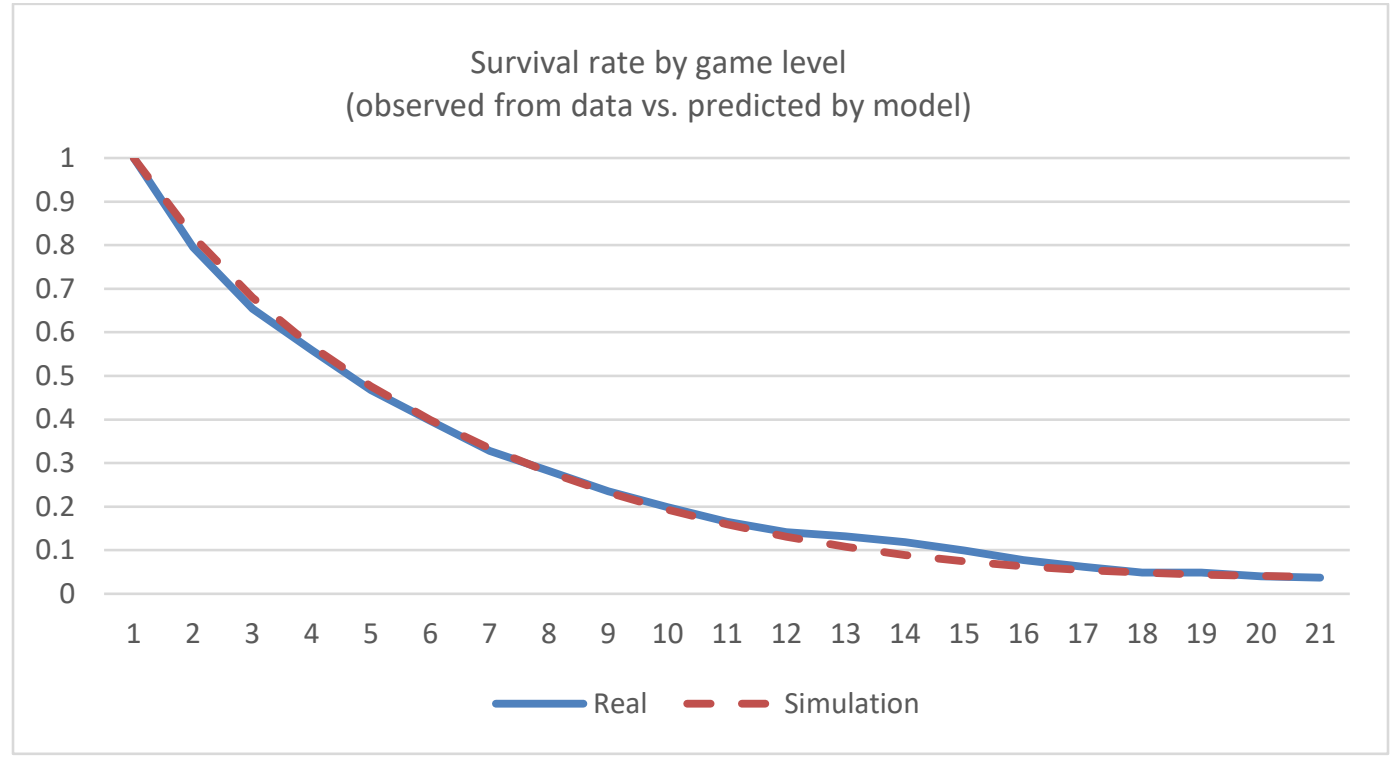

Figure $2 b$. Simulated survival rate by game level and by class

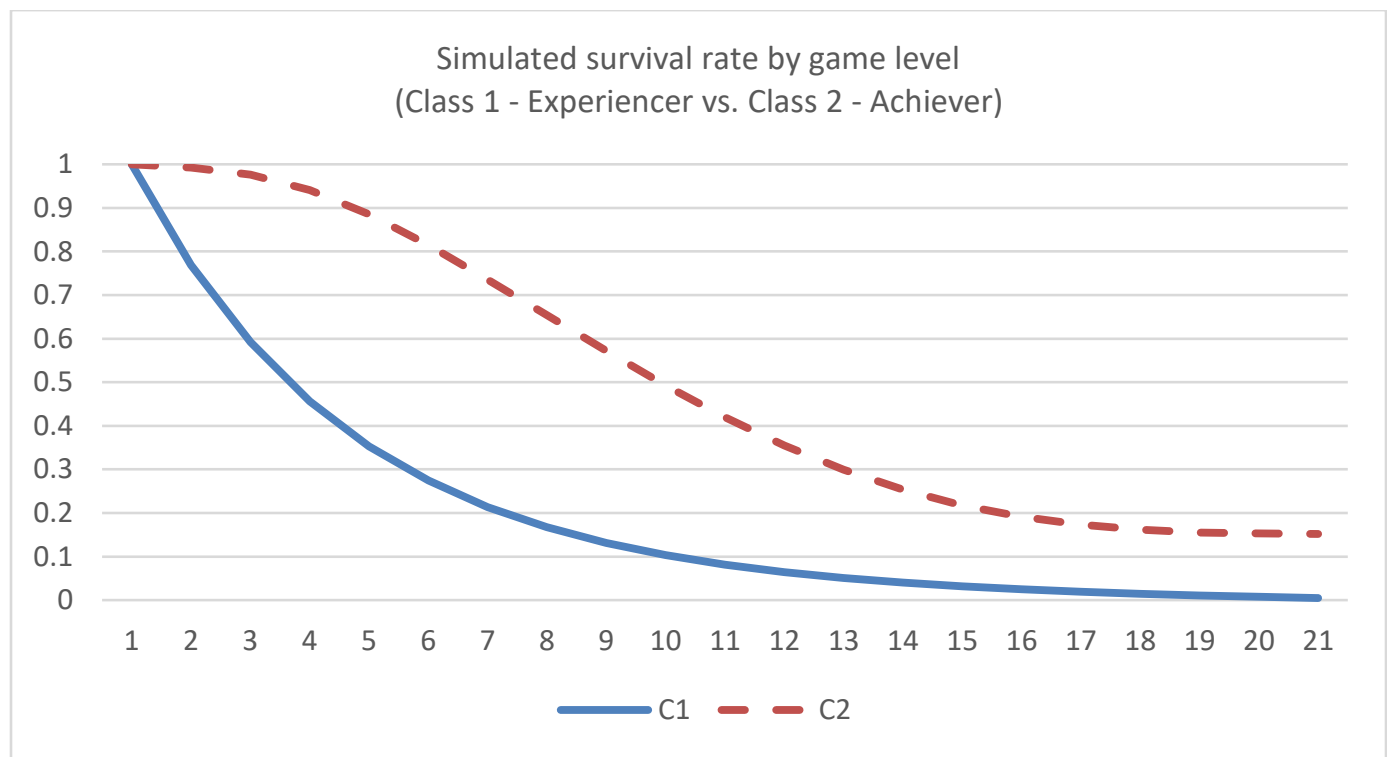


Figure 3a. Average gain from operation over game level over game levels

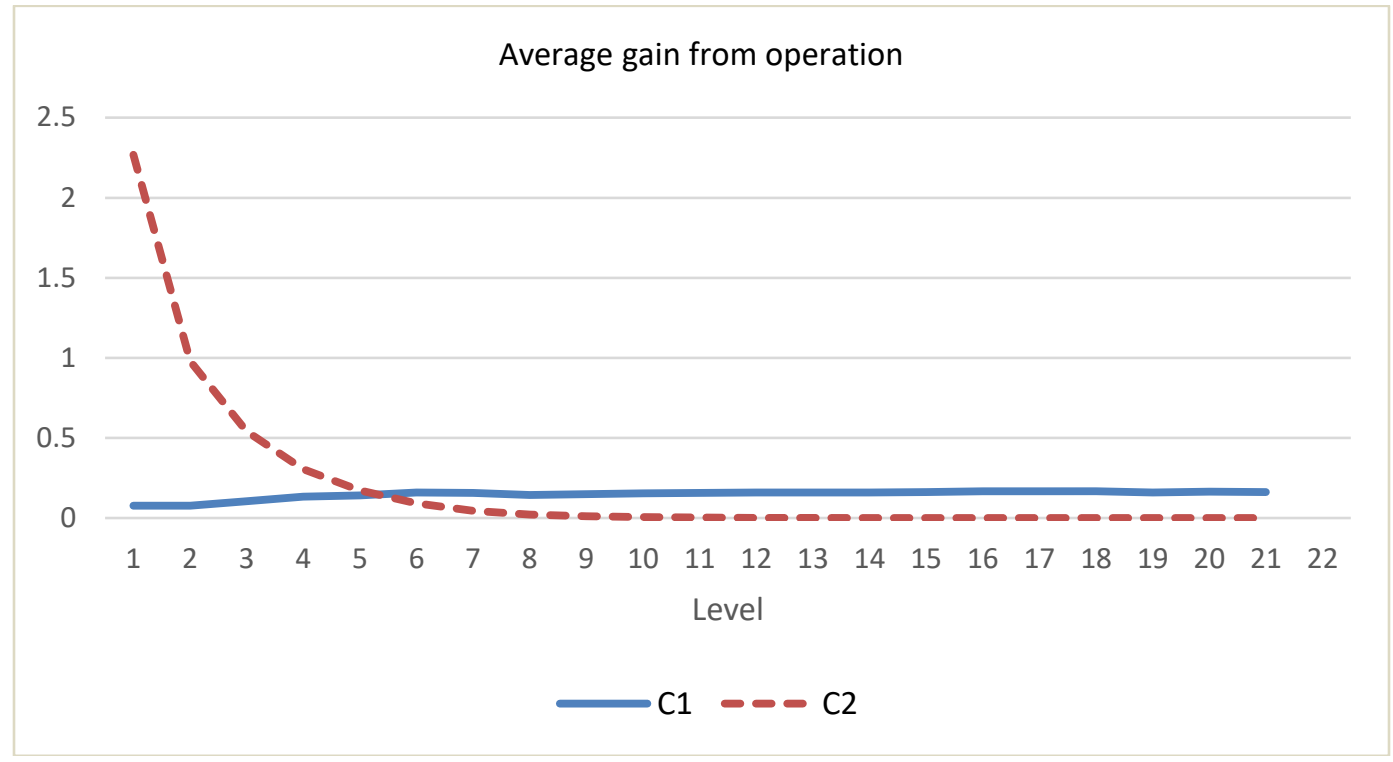

Figure 3b. Average loss from operation over game levels

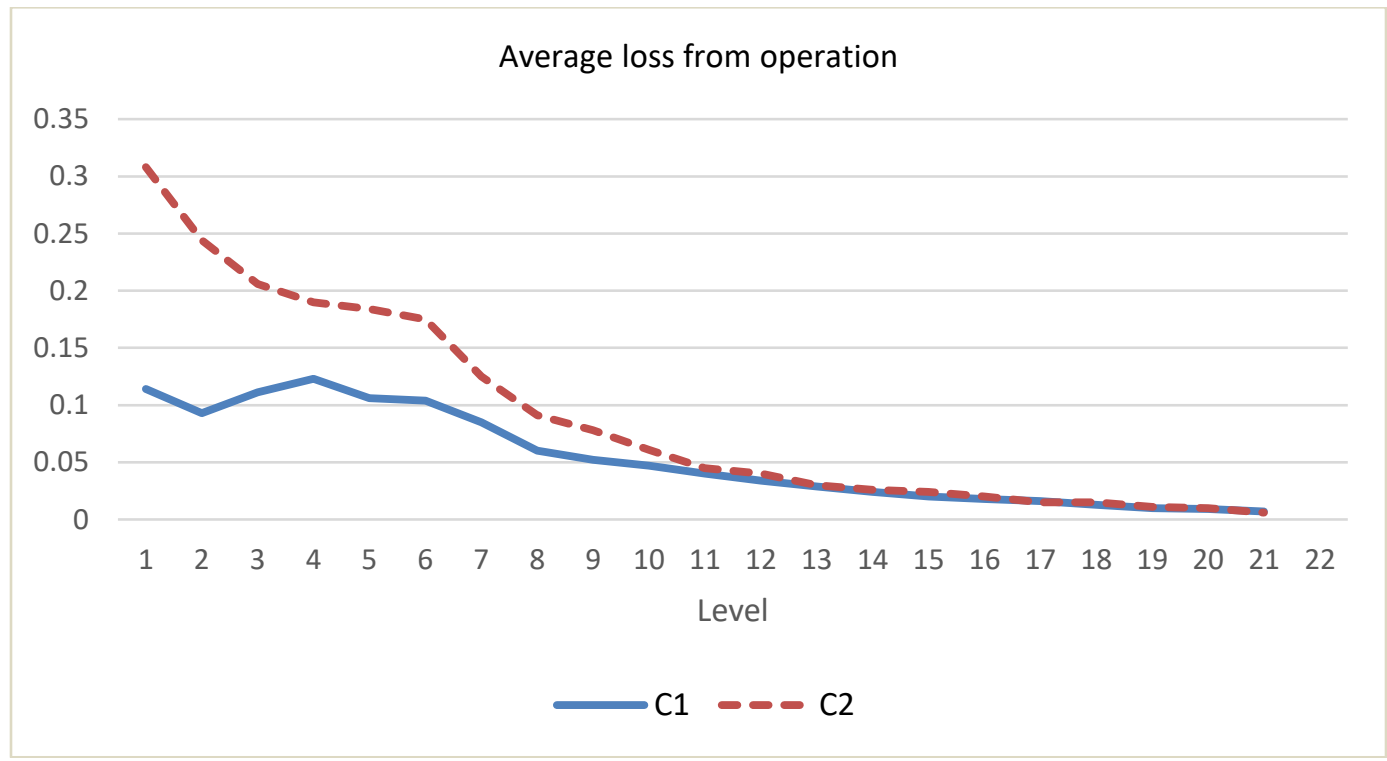


Figure 3c. Average SOA over game levels

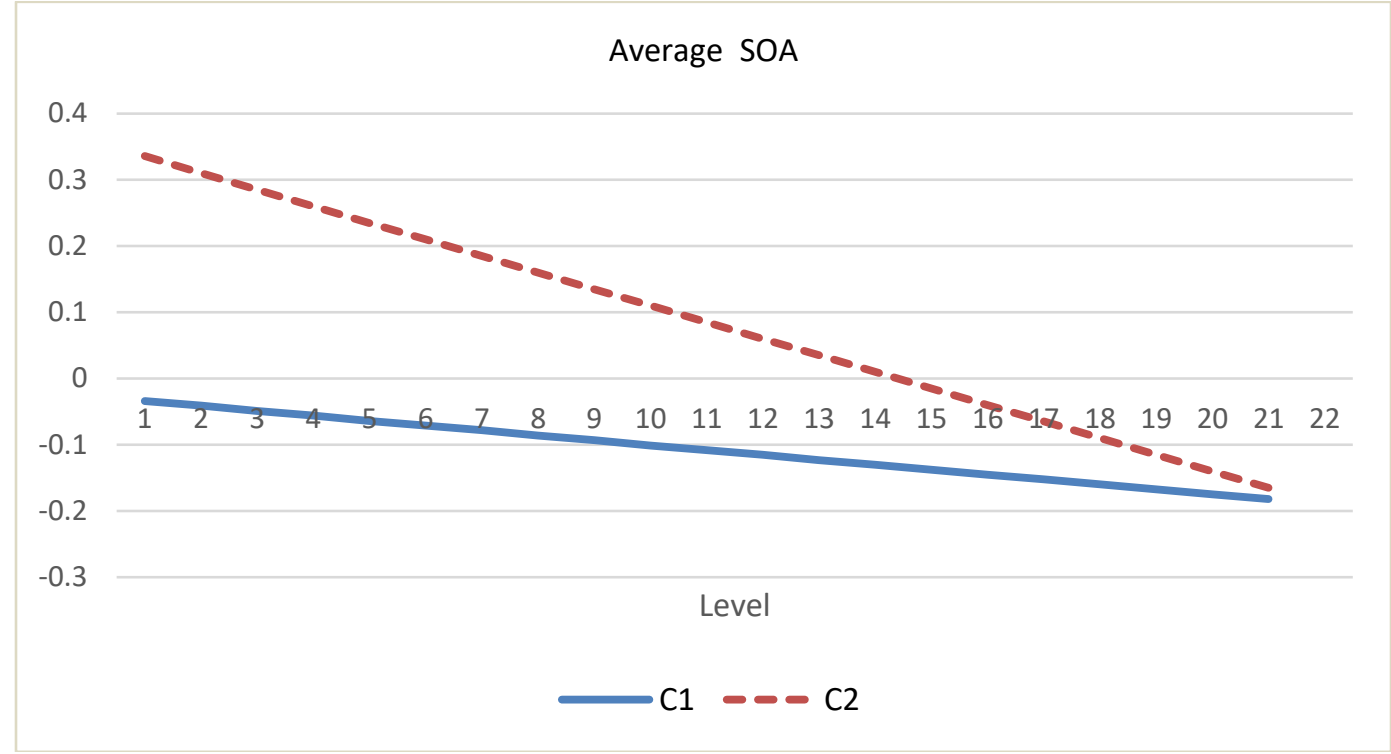

Figure 3d. Average future value over game levels

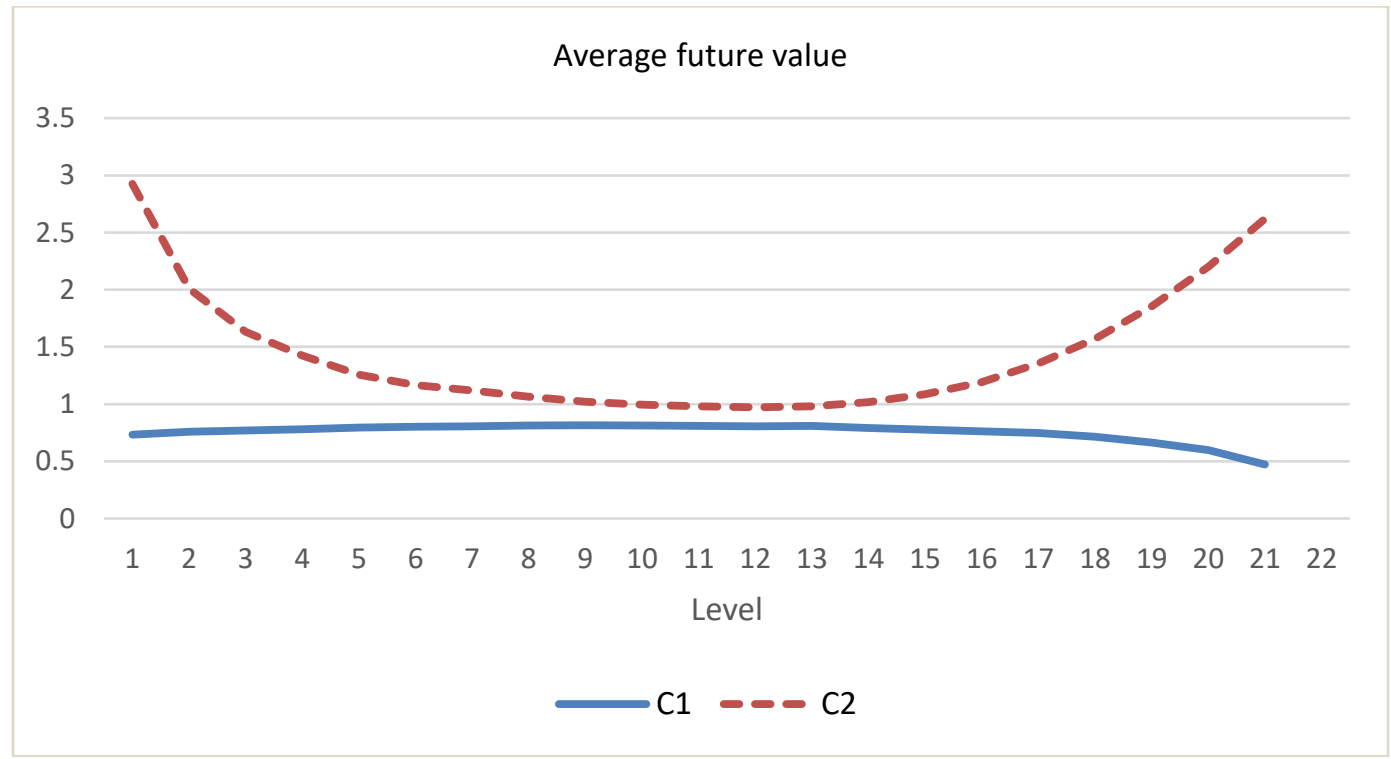


Figure 4a. Average of the individual perceived mean for the intercept over game levels

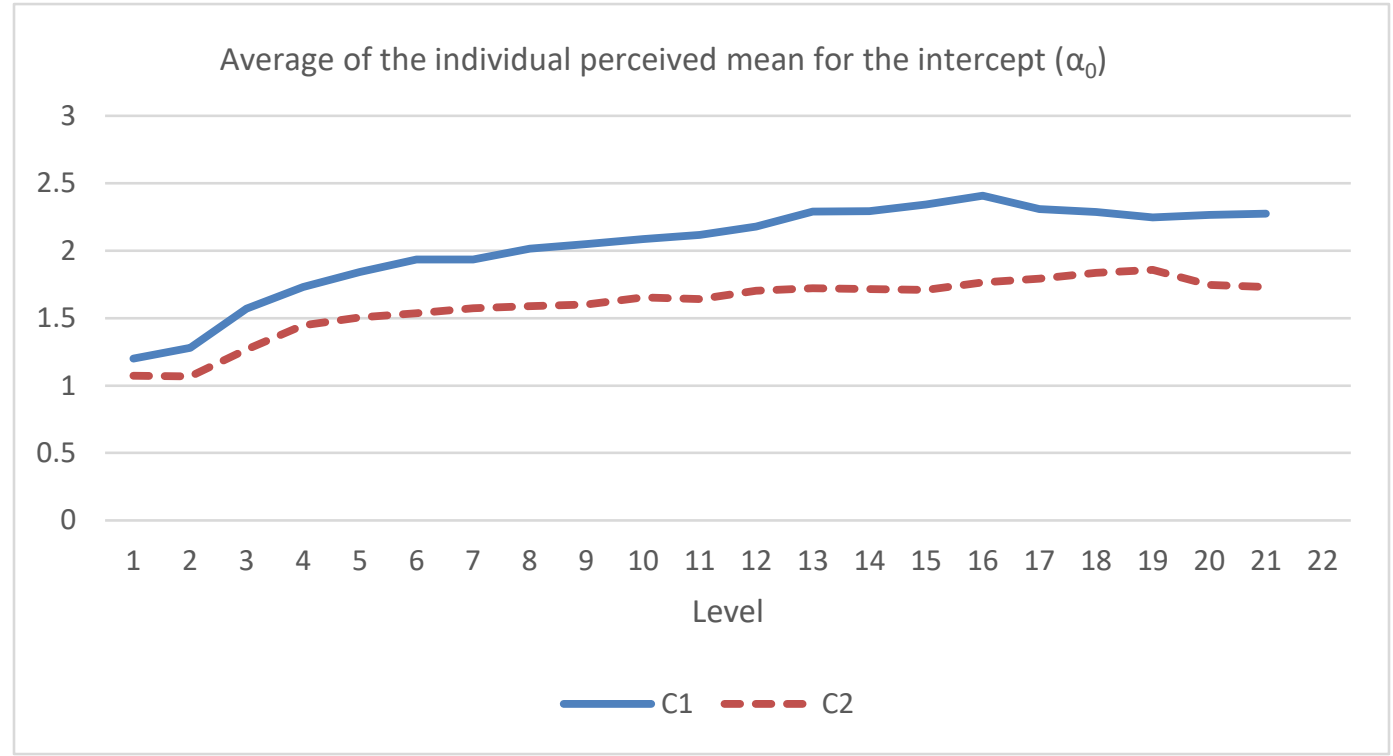

Figure 4b. Average of the individual perceived mean for the slope over game levels

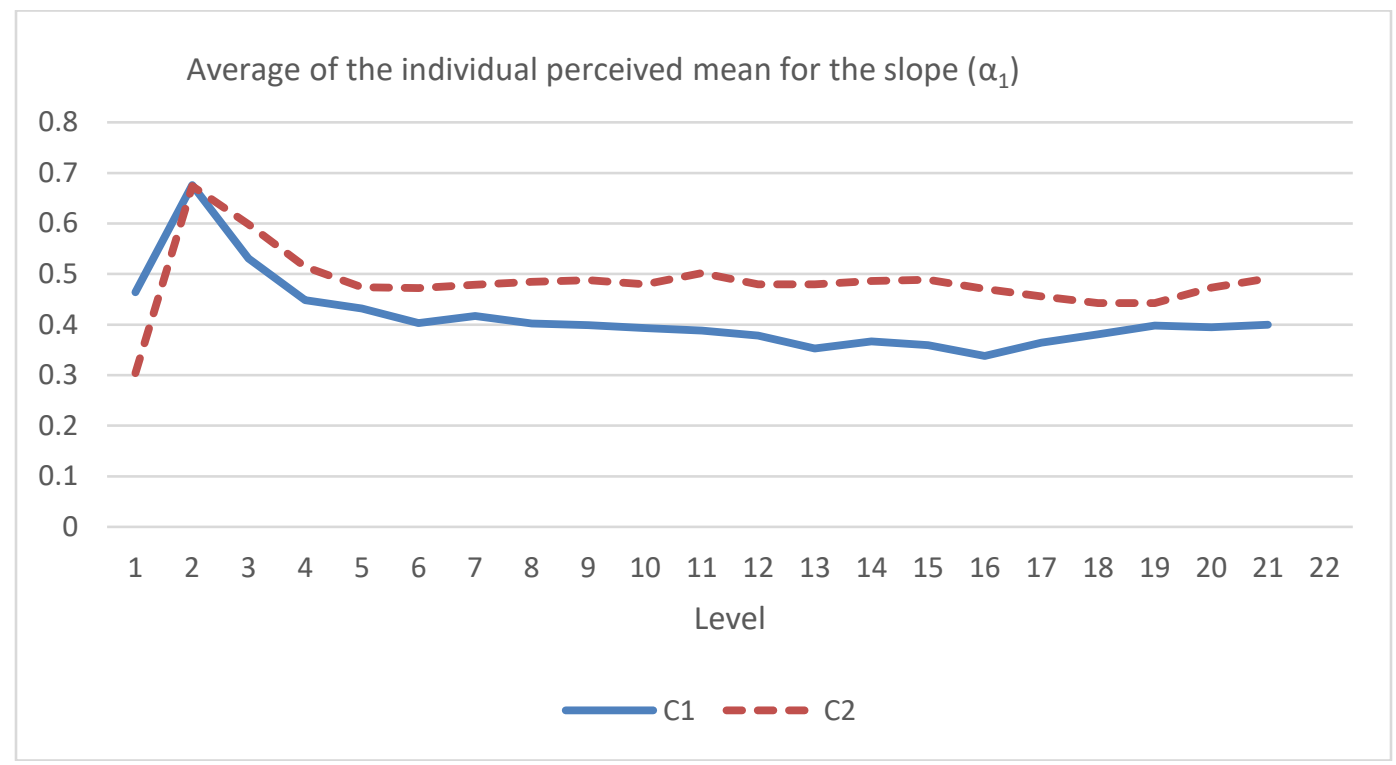


Figure 4c. Average of the individual perceived variance for the intercept over game levels

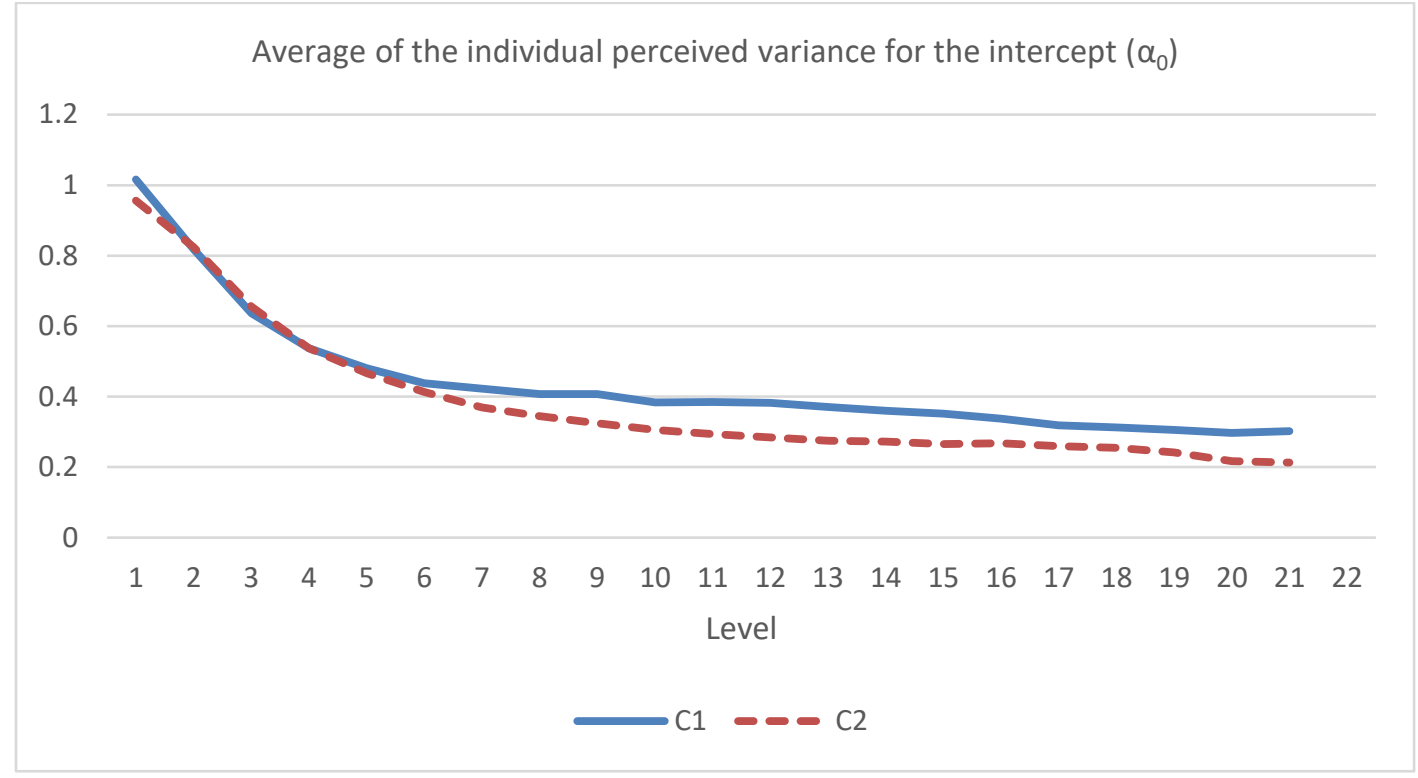

Figure 4d. Average of the individual perceived variance for the slope over game levels

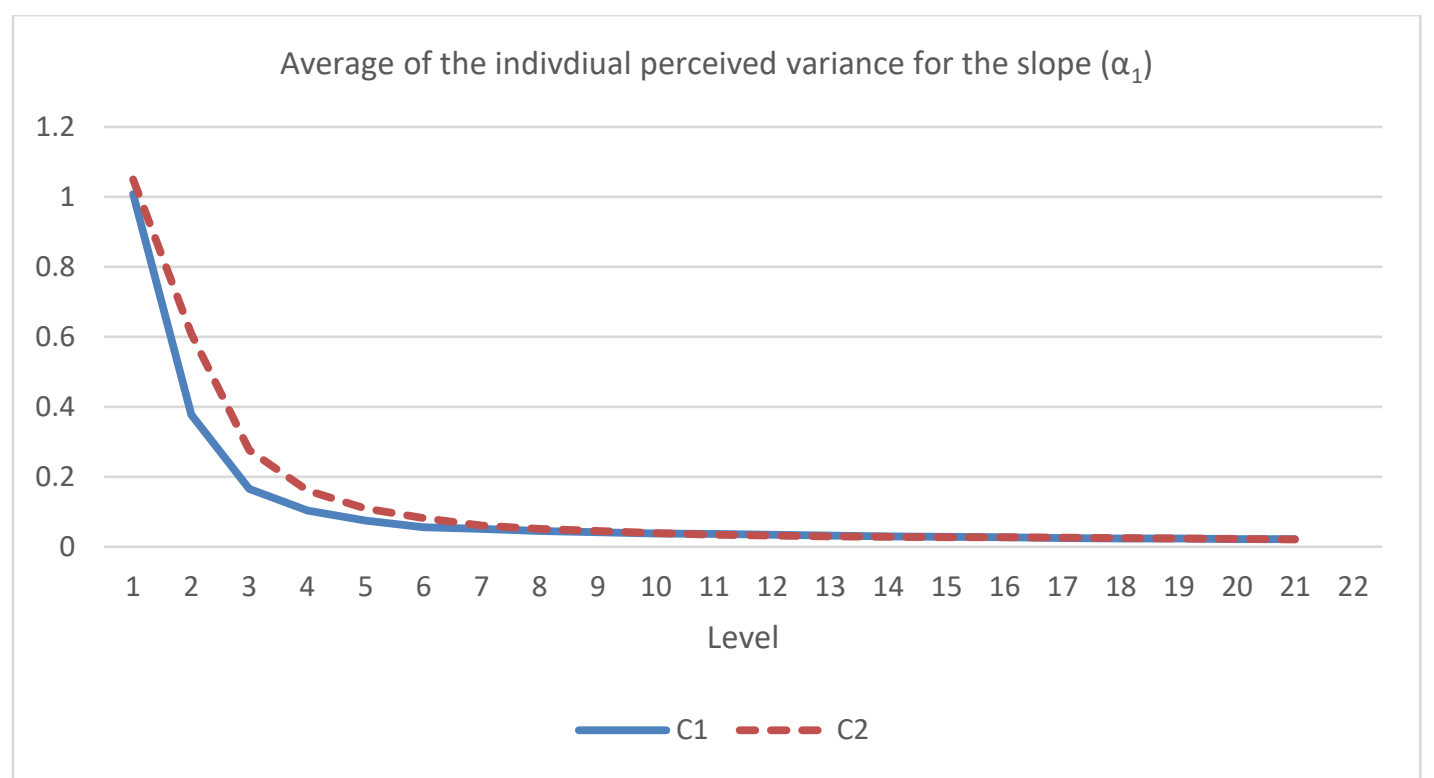


Figure 5a. Cumulative required passing point designs

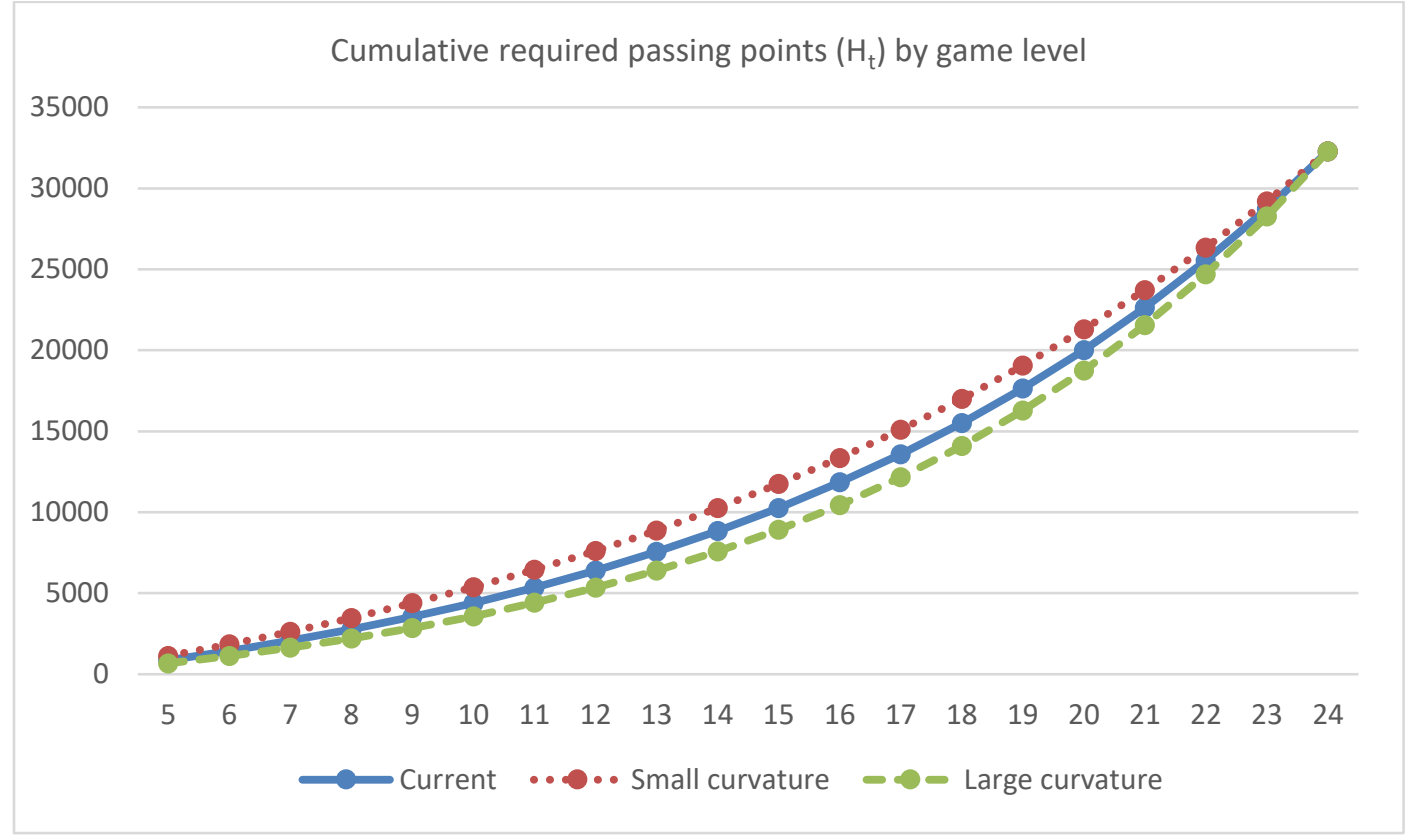

Figure 5b. Incremental required passing point designs

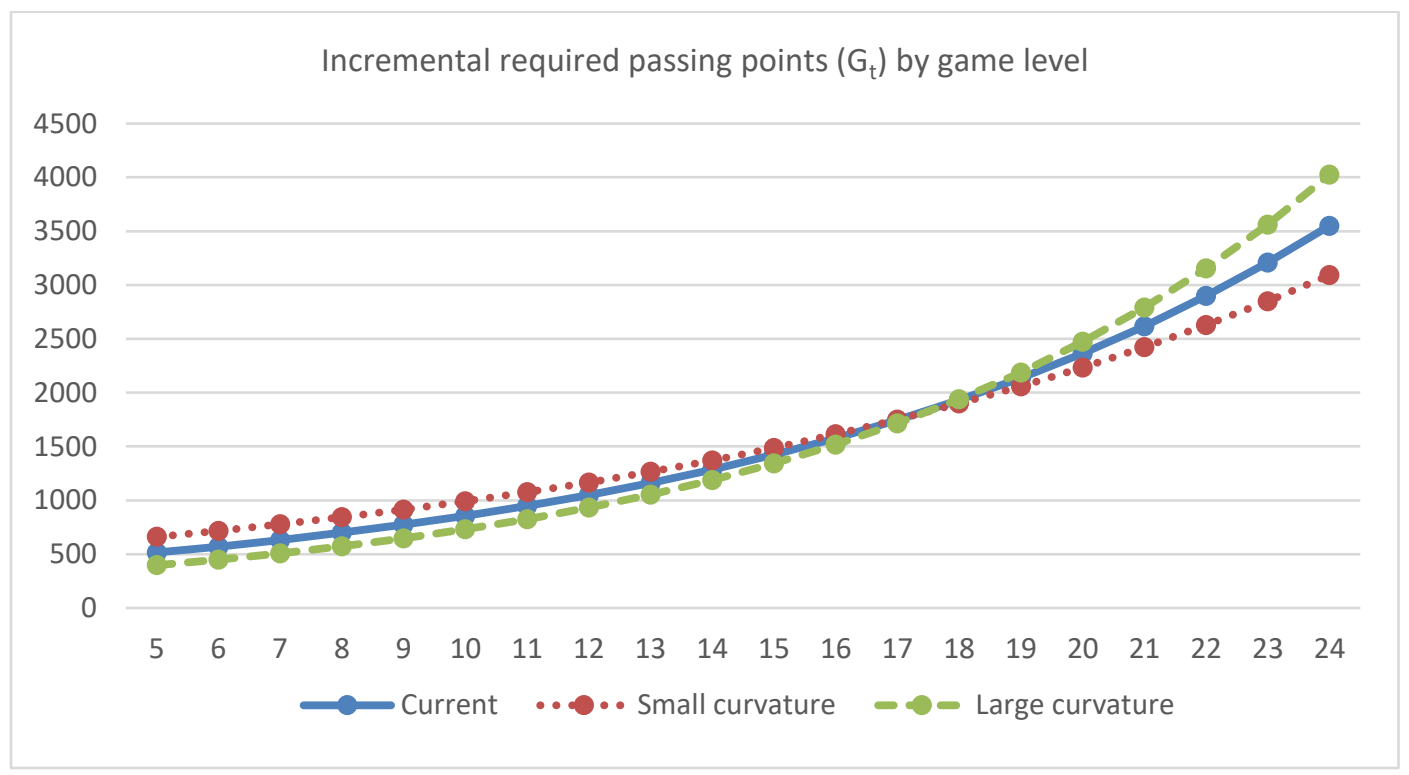

